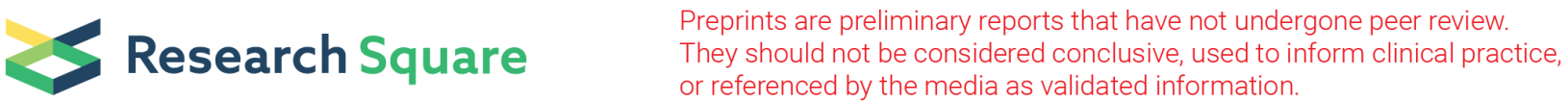

\section{Ablation of Histone Demethylase KDM5B in Melanoma Augments Anti-Tumor Immunity through Upregulation of Retroelements}

Shang-Min Zhang

Yale School of Medicine

Wesley Cai

Yale University School of Medicine

Xiaoni Liu

University of lowa

Durga Thakral

Yale University

Goran Micevic

Yale University

\section{Carmen Booth}

Yale University School of Medicine https://orcid.org/0000-0002-2153-738X

Kim Blenman

Yale University

Lucia Jilaveanu

Yale University

William Damsky

Yale University https://orcid.org/0000-0003-0975-4071

Meaghan McGeary

Yale School of Medicine https://orcid.org/0000-0002-8505-7158

Mario Sznol

Yale University

Harriet Kluger

Yale School of Medicine

Akiko Iwasaki

Yale University https://orcid.org/0000-0002-7824-9856

Marcus Bosenberg

Yale University School of Medicine

Qin Yan ( $\nabla$ qin.yan@yale.edu )

Yale University https://orcid.org/0000-0003-4077-453X 


\section{Biological Sciences - Article}

Keywords: Epigenetic therapies, anti-tumor T cell immunity, up-regulation of retroelements, tumor immunogenicity

Posted Date: November 6th, 2020

DOI: https://doi.org/10.21203/rs.3.rs-87705/v1

License: (c) (i) This work is licensed under a Creative Commons Attribution 4.0 International License. Read Full License

Version of Record: A version of this preprint was published at Nature on October 20th, 2021. See the published version at https://doi.org/10.1038/s41586-021-03994-2. 


\section{Abstract}

Epigenetic therapies are emerging strategies to prime host immune system to immunotherapies. Here we show that depletion of H3K4 demethylase KDM5B in melanoma cells induces anti-tumor T cell immunity through up-regulation of retroelements, which activates cytosolic nucleic acid sensing pathways and subsequent type I interferon responses. Mechanistically, KDM5B recruits H3K9 methyltransferase SETDB1 to the chromatin to repress these retroelements. Ablation of KDM5B enhances responses of poorly immunogenic melanoma tumors to anti PD-1 treatment. Our studies suggest that therapies targeting KDM5B are a novel approach to enhance tumor immunogenicity and overcome immunotherapy resistance.

\section{Main}

KDM5B expression is negatively correlated with anti-tumor immunity and response to immune checkpoint blockade. To explore the roles of KDM5B in melanoma, we performed Gorilla Gene Ontology analysis of 9,336 genes negatively correlated with KDM5B expression in TCGA melanoma dataset. The top 5 gene ontology categories anti-correlated with KDM5B expression are all related to immune system processes (Fig.1a). To further examine the relationship of KDM5B with anti-tumor immune responses, we correlated KDM5B mRNA levels with T cell markers, antigen presentation and cytokine genes in TCGA melanoma dataset. Remarkably, KDM5B expression is inversely associated with all the genes examined, including IFNG (IFN- $\gamma$ ) and TNF (TNF-a), two crucial effector cytokines in anti-tumor immunity ${ }^{1}$ (Extended Data Table 1). These results suggest that KDM5B expression is inversely correlated with intra-tumoral inflammation. Consistently, patients with higher KDM5B expression have lower expression of markers for $\mathrm{CD}^{+} \mathrm{T}$ cells in the tumors in TCGA melanoma dataset (Extended Data Fig. 1a). Lack of $\mathrm{T}$ cell infiltration

in pre-treatment biopsies normally correlates with resistance to immune checkpoint blockade (ICB) ${ }^{2}$. To evaluate whether KDM5B levels predict response to immune checkpoint blockade in melanoma, we analyzed RNA-seq data of pre-treatment specimens from tumors sampled prior to anti-PD-1 therapy ${ }^{3}$. We found that KDM5B expression levels are significantly lower in patients with complete response than those in patients with progressive disease (Fig.1b and Extended Data Table 2). Furthermore, we evaluated KDM5B protein levels in a tissue microarray (TMA) containing samples from patients treated with ICB. Patients who responded to ICB had a very low intensity of KDM5B staining and lacked detectable KDM5B ${ }^{\text {high }}$ melanoma cells, while the non-responders had a major subset of KDM5B ${ }^{\text {high }}$ melanoma cells (Extended Data Fig. 1b). These data raised the possibility that KDM5B could be a biomarker for poor response to ICB and a KDM5B targeting therapy might overcome resistance to ICB.

\section{KDM5B loss induces anti-tumor immunity in a murine model of melanoma}

To test whether ablation of KDM5B in melanoma induces anti-tumor immunity, we assessed the effects of $K d m 5 b$ deletion in the immunogenic YUMMER1.7 mouse melanoma cells ${ }^{4}$ on their tumor growth in syngeneic C57BL/6J mice (Fig. 1c, and Extended Data Fig. 2a). Polyclonal Kdm5 $b^{-/-}$YUMMER1.7 cells were rejected completely after subcutaneous implantation of 500,000 cells; whereas all wild type 
YUMMER1.7 injections formed tumors as expected. Furthermore, the mice that rejected $K d m 5 b^{-/-}$tumors are protected against re-challenge by wildtype control tumor cells, suggesting the development of immune memory. We then compared the tumor microenvironment of $K d m 5 b^{-1-}$ YUMMER1.7 bearing mice (prior to complete tumor rejection) with the control. KDM5B loss results in a significant increase in CD $8^{+} T$ cells (Fig. 1d) as well as total T cells in these tumors, as demonstrated using flow cytometry and immunohistochemistry (Fig 1d, Extended Data Fig. 2b-d). We detected increased apoptosis in the KDM5B knockout tumors, as detected by cleaved caspase 3 immunohistochemistry (Extended Data Fig. 2d). Consistent results were obtained with $K d m 5 b^{-/-}$YUMMER1.7 single cell-derived clones (Extended Data Fig. 2e). These differences are diminished in immunodeficient Rag $^{1 /-}$ mice, which lack both $\mathrm{B}$ and $\mathrm{T}$ cell activity (Fig.1e). Taken together, these results demonstrate that KDM5B depletion induces anti-tumor immunity that is dependent on the adaptive immune system.

\section{KDM5B loss overcomes resistance to ICB in a murine melanoma model}

Immune checkpoint blockade therapies can induce long lasting anti-tumor responses in a subset of patients. However, there are other patients that have either primary or acquired resistance to checkpoint blockades ${ }^{5}$. We next examined the effects of KDM5B loss on a ICB resistant murine melanoma model. YUMM1.7 is a poorly immunogenic mouse melanoma cell line, and does not respond to anti-PD-1 treatment ${ }^{4}$. Depletion of KDM5B in YUMM1.7 not only significantly decreases the growth rate of YUMM1.7 tumors, but also renders these cells sensitive to anti-PD-1 treatment (Fig.1e, Extended Data Fig 2f). These results suggest the KDM5B loss could overcome resistance to anti-PD-1 treatment.

\section{KDM5B loss activates the type I interferon pathway}

We performed RNA-seq analysis of $K d m 5 b^{-/-}$and control YUMMER1.7 cells (Extended Data Table 3). Gene Set Enrichment Analysis (GSEA) showed that genes in the activated type I interferon pathways, such as RIG-I like receptor signaling and cytosolic DNA sensing pathways, are elevated in $K d m 5 b^{-/}$cells (Fig. 2a). Consistently, KDM5B depletion led to increased protein levels of MDA5, RIG-I, MAVS, cGAS, IRF9, and phosphorylated TBK1, IRF3, and STAT1 (Fig.2b). Similarly, in other mouse melanoma cell lines, Ifngr T/- $^{-1}$ YUMMER1.7 cells, YUMM1.7, and 1445 (Cdkn2a and Pten inactivated with $\beta$-catenin stabilization) ${ }^{6}$, we also observed activation of interferon stimulated genes (ISGs) by $K d m 5 b$ depletion using sgRNAs or shRNA (Extended Data Fig 3a-c). To identify whether innate RNA or DNA sensing pathways contributed to the activated type I interferon pathway in $K d m 5 b^{-1-}$ cells, we further depleted RNA or DNA sensors in $K d m 5 b^{-1-}$ YUMMER1.7 cells and examined their impacts on ISGs induction and tumor growth ability. Depletion of MDA5 or cGAS in $K d m 5 b^{-/-}$YUMMER1.7 cells, significantly diminished induction of ISGs (Fig. 2c) and partially rescued their ability to form tumors in vivo (Fig. 2d), while depletion of RIG-I (encoded by Ddx58 gene) did not affect induction of ISGs (Fig 2c). Similar results were observed in YUMM1.7 and 1445 mouse melanoma cell lines (Extended Data Fig 3d-g). The fact that RNA sensor MDA5 but not RIG-I is critical for induction of ISGs, suggests that long dsRNAs are likely critical for activation of type I IFN by $K d m 5 b$ loss in mouse melanoma cells, instead of ssRNAs or short dsRNAs, which are preferred 
substrates for RIG-I ${ }^{7}$. Consistently, abrogation of downstream adaptor MAVS for RNA sensing or STING (encoded by Sting 1 gene) for DNA sensing in $K d m 5 b^{-/-}$cells, also partially rescued their tumor forming ability in vivo (Fig. 2d). Moreover, simultaneous depletion of both MAVS and STING further rescued in vivo tumor growth phenotype of $K d m 5 b^{-/-}$cells (Fig. $2 \mathrm{~d}$ ). These results suggest that both RNA and DNA sensing pathways are critical for the activation of type I interferon pathway induced by KDM5B depletion. In addition, ablation of KDM5B increases the level of MHC class I molecule expression as well as genes involved in antigen processing and presentation pathways in mouse melanoma cell YUMMER1.7, YUMM1.7 and YUMM3.3 (Extended Data Fig 4a-c).

\section{KDM5B loss induces reactivation of retroelement expression}

Bidirectional transcription of endogenous retroelements induces formation of "non-self" dsRNAs, which can activate host pattern recognition receptors and initiate type I interferon responses ${ }^{8}$. Stranded specific RNA-seq analysis of repetitive elements found increased transcripts from retroelements in both sense and antisense directions, including Long Terminal Repeat (LTR) containing Endogenous RetroViruses (ERVs) as well as non-LTR elements, such as Long Interspersed Nuclear Elements (LINEs) in Kdm5 $\mathrm{b}^{-/-}$ YUMMER1.7 cells compared to control YUMMER1.7 cells (Fig. 3a and 3b, and Extended Data Table 4 and 5). $K d m 5 b$ inactivation also led to induction of retroelements in YUMM1.7 and 1445 mouse melanoma cells (Extended Data Fig 5a-b). These induced retroelements led to global increases of dsRNAs in YUMMER1.7 and 1445 cells (Extended Data Fig 5c-d). As our data also showed that DNA sensing pathways contribute to the activation of type I IFN signaling, we reasoned that dsDNAs may be generated via reverse-transcription of retroelements and thereby contribute to DNA sensing pathway-induced type I interferon signaling. To test this possibility, we assessed the effects of a combination of reverse transcriptase inhibitors (RTis) Zidovudine and Nevirapine on $\mathrm{Kdm} 5 \mathrm{~b}^{-/-}$cells. Consistent with our hypothesis, this treatment suppressed activation of type I IFN pathway (Fig. 3c). Furthermore, we observed decreased cytosolic retroelement cDNAs in RTi treated $K d m 5 b^{-/-}$cells (Fig. $3 d$ ), while RTi treatment had no effect on the copy numbers of mitochondrial DNA (Fig.3e). MMVL30 is among the top induced ERVs by KDM5B depletion. To directly test the role of highly induced MMVL30 on induction of ISGs, we depleted MMVL30 using either transient transfection of siRNA or a stable lentiviral shRNA system. Down-regulation of MMVL30 decreased ISG induction in both systems (Fig.3f-g), suggesting that MMVL30 contributes significantly to KDM5B loss-induced ISG induction.

\section{KDM5B directly repress retroelements in a demethylase-independent manner}

We then assessed whether the retroelements induced after KDM5B loss are direct targets of KDM5B. KDM5B ChIP-qPCR analysis on representative retroelement loci in YUMMER1.7 showed that these loci are bound by KDM5B (Fig.4a). Similarly, KDM5B ChIP-seq analysis showed enrichment of KDM5B binding to ERVs derepressed in $K d m 5 b^{-/-} 1445$ mouse melanoma cells (Extended Data Fig 6 a,b and Extended Data Table 6,7). Surprisingly, H3K4me3 levels on these KDM5B bound loci were not altered upon KDM5B depletion (Fig.4b), suggesting that KDM5B does not affect the expression of retroelements with its H3K4me3 demethylase activity. Consistently, re-introduction of either wild-type KDM5B or enzymatic dead 
mutant KDM5B into $\mathrm{Kdm} 5 \mathrm{~b}^{-/}$cells repressed the induced expression of retroelements and ISGs (Fig. 4c), and rescued the tumor growth defects of these cells in vivo to the same extent (Fig.4d). Consistently, treatment with pan-KDM5 inhibitors CPI-48 or KDM5-C70 did not significantly induce retroelements or ISGs (Extended Data Fig 7a-d). Together, these data suggest that the regulation of retroelements by KDM5B is independent of its demethylase activity.

Since there is no increase of active H3K4me3 mark, we hypothesized that a decrease in repressive histone marks could contribute to the phenotype. To identify which repressive marks are responsible, we treated YUMMER1.7 cells with either H3K27me3 methyltransferase inhibitors (EZP6438 and GSK343) or the H3K9me3 methyltransferase inhibitor chaetocin (Extended Data Fig. 8a,b). While H3K27me3 methyltransferase inhibitors had minimal effects, $\mathrm{H} 3 \mathrm{~K} 9$ me3 methyltransferase inhibitor induced the expression of MMVL30 and ISGs in a dose-dependent manner (Extended Data Fig. 8a,b). Consistent with these results, we did not observe significant changes of H3K27me3 levels at the MMVL30 and RLTR6 (Extended Data Fig. 8c). In contrast, ChIP-qPCR showed decreased H3K9me3 levels on those retroelement loci in $\mathrm{Kdm} 5 \mathrm{~b}^{-/-}$cells (Fig.4e).

Next, in order to assess which H3K9me3 methyltransferase is the potential modulator, we deleted G9a, Suv39h1 or Setdb1 in YUMMER1.7 cells (Extended Data Fig. 8d-f) and compared the levels of ERVs and ISGs in each with those in the control cells. Setdb1 loss led to significant induction of MMVL30, while G9a and Suv39h1 deletion had no effect on ERVs including MMLV30 and ISGs (Extended Data Fig. 8f-g). Similar regulation of MMVL30 by SETDB1 has been reported in mouse embryonic fibroblasts (MEF) ${ }^{9}$. We then compared SETDB1 binding on those retroelement loci in WT cells with GFP control, $K d m 5 b^{-/-}$with GFP control, $K d m 5 b^{-/}$cells re-introduced with WT KDM5B or enzymatic dead KDM5B. SETDB1 binding on those retroelements was reduced in $K d m 5 b^{-/}$cells compared with control cells, and was partially restored when either WT KDM5B or enzymatic dead KDM5B was re-introduced (Fig.4f).

To explore the mechanism by which KDM5B regulates SETDB1 binding on those retroelement loci, we asked whether KDM5B forms a complex with SETDB1. Co-immunoprecipitation (IP) using YUMMER1.7 nuclear extracts showed that endogenous SETDB1 IP pulls down KDM5B, suggesting that they do indeed form a complex in the nucleus (Fig.4g). Similarly, this interaction was observed by IP of either endogenous SETDB1 or KDM5B from nuclear extracts of 1445 and YUMM1.7 mouse melanoma cells, as well as MC38 mouse colorectal cancer cells (Extended Data Fig.9a-b). Furthermore, we noticed that there is a decreased nuclear level of SETDB1 in $K d m 5 b^{-1-}$ cells (Fig. $4 \mathrm{~g}$ ). We found that treatment with the proteasome inhibitor MG132 significantly increased nuclear SETDB1 protein levels in $K d m 5 b^{-1-}$ YUMMER1.7 cells (Extended Data Fig 9c), which suggests KDM5B also regulates SETDB1 protein stability in the nucleus in a proteasome-dependent manner.

To further test whether the protein level of SETDB1 or recruitment of SETDB1 to the retro-elements is critical for the phenotype, we overexpressed either WT or enzymatic mutant SETDB1 in $K d m 5 b^{-/-}$ YUMMER1.7 cells and assessed their impact on the expression of retro-elements and ISGs. Neither WT 
nor mutant SETDB1 repressed induction of retroelements or ISGs in $K d m 5 b^{-1-}$ YUMMER1.7 cells (Extended Data Fig 9d-e). Consistently, H3K9me3 ChIP-qPCR analysis of those retroelements showed that overexpression of SETDB1 in $K d m 5 b^{-/}$cells is unable to increase H3K9me3 binding, while either WT or enzymatic dead mutant KDM5B increases H3K9me3 binding on those loci (Extended Data Fig 9f).

Furthermore, SETDB1 was unable to fully rescue the ability of $K d m 5 b^{-/-}$YUMMER1.7 cells to form tumors in vivo (Extended Data Fig 9g). These data suggest that KDM5B recruits SETDB1 to increase H3K9me3 levels at the retroelements, represses expression of these retroelements and ISGs, and promotes tumor growth (Fig. 4h). It is worth mentioning that in embryonic stem cells, $66 \%$ of regions co-marked by H3K9me3/H3K4me3 are enriched with LINE elements and ChIP-X enrichment analysis suggesting that SETDB1 and KDM5B binds to these marks ${ }^{10}$.

\section{KDM5B represses ERVs and ISGs in human melanoma.}

Consistent with our results in mouse melanoma cells, inactivation of KDM5B in human melanoma cell YUDOSO and YURIF cell lines also induced expressions of ERVs and ISGs (Extended Data Fig 10a-b). In YUDOSO human melanoma cells, RNA sensing pathway genes DDX58 and MAVS are critical for activation of the type I IFN pathway, but DNA sensing pathway genes CGAS and STING1 are not (Extended Data Fig 10c). In YUDOSO cells, RIG-I but not MDA5 deletion rescued ISG induction by KDM5B knockdown (Extended Data Fig 10c), which suggests ERVs induced in YUDOSO human melanoma cells could potentially form single stranded RNAs or short dsRNAs. We further analyzed the published transcriptome data of pre-anti-PD-1 treatment melanoma biopsies ${ }^{3}$ and found that the ERVs anticorrelated with KDM5B expression such as ERVmap_2637 are expressed significantly higher in patients with complete responses to anti-PD-1 treatment than patients with progressive disease (Extended Data Fig 11a-c). In contrast to KDM5B, KDM5A, KDM5C or KDM5D were not significantly anti-correlated antiPD-1 response (compare Fig. 1b with Extended Data Fig 11d-f). We also analyzed several known immunemodulatory epigenetic regulators, KDM1A, DNMT1 and DNMT3B (Extended Data Fig 11g-i). Consistent with the previous reports ${ }^{11-13}$, they are also anti-correlated with anti-PD-1 responses. In comparison, there is no significant correlation for EZH2 (Extended Data Fig 11j).

Here we show that KDM5B loss in melanoma cells de-represses expression of endogenous retroelements, activates type I IFN signaling pathway and increases $\mathrm{T}$ cell infiltration. The enhanced anti-tumor immunity from KDM5B loss augments responses to immune checkpoint blockade and overcomes the resistance to immunotherapy. These results suggest that therapies targeting KDM5B with KDM5B degraders can improve efficacy and overcome resistance to immune checkpoint blockade.

\section{Methods}

\section{Cell culture}

Stable KDM5B knockout (KO) and further DNA or RNA sensor KO (RIG-I, MDA5,CGAS, MAVS an STING) cells in mouse melanoma lines YUMMER1.7, YUMM1.7, 1445 or human melanoma cell line YURIF and 
YUDOSO (obtained from Dr. Ruth Halaban's lab at Yale University) were generated using lentiCRISPR-v2 system and selected with puromycin or blasticidin as previously described ${ }^{14}$. For validation, KDM5B KO with or without DNA or RNA sensors double KO or triple KO non-virus single clones of YUMMER1.7 were generated with pSpCas9 (BB)-2A-GFP(pX458). Stable knockdowns of KDM5B in mouse or human melanoma cells were generated with lentiviral shRNA hairpins ${ }^{14}$. Doxycyline-inducible knockdown of MMVL30 in Kdm5b KO cell was generated with pINDUCER10 shRNA constructs. pLX304 3XHA-KDM5B or Flag-SETDB1 lentiviral vectors were constructed by gateway cloning. Stable cell lines were generated using pLX304 vectors and selected with blasticidin. SgRNA, shRNA and siRNA information are listed in Extended Data Table 8,9.

\section{Animal Experiments}

Male C57BL/6J and C57BL/6JRag1 $1 /-$ mice were purchased from Jackson laboratories. All mouse procedures were approved by the Institutional Animal Care and Use Committee of Yale University. Five to seven-week-old mice were age matched for tumor inoculation. For YUMMER1.7 cell, $0.5 \times 10^{6}$ cells in $100 \mu l$ PBS were injected. For YUMM1.7 cell, 250,000 cells were injected. For immunotherapy treatments, antiPD-1 antibody (RMP1-14) and Rat IgG (28956) were purchased from Bio X cell. Mice were treated with $100 \mu \mathrm{g}$ anti-PD-1 or Rat IgG twice per week for 3 weeks since day 7 after tumor injection for YUMM1.7. Tumor volumes were calculated using the equation: $0.5233^{*} \mid * W^{*} \mathrm{~h}$.

\section{Immunohistochemistry}

Immunohistochemical (IHC) sections and staining were done by Yale Mouse Research Pathology and Department of Pathology Research Histology. Anti-cleaved caspase 3 (Abcam \#ab4051) and anti-CD8 antibody (Abcam \#ab217344) were used. Images of representative fields were taken.

\section{Flow cytometry analysis}

On day 14 or 15 after tumor injection, tumors were dissected, minced and digested with $1 \mathrm{mg} / \mathrm{ml}$ Collagenase/Dispase (Sigma-Aldrich 10269638001) and 1 X DNase I (Qiagen 79254) at $37^{\circ} \mathrm{C}$ for 30 minutes. Dissociated tumor cells were filtered through a $70 \mu \mathrm{m}$ filter to achieve a single-cell suspension before staining for flow cytometry analysis. Cells were stained using anti-CD45 APC-Cy7 (BioLegend 103115) or anti-CD45 Pacific Blue (BioLegend 103126), anti-CD3 FITC (BD Bioscience 553062), anti-CD8 PerCP/Cy5.5 (BioLegend,100733), anti-H-2Kb/H-2Db FITC (BioLegend, 114605), and Live/Dead Fixable Red Dead CellStain Kit (ThermoFisher Scientific L34971) for 30 minutes at $4{ }^{\circ} \mathrm{C}$ in the dark. Flow cytometry was performed using a BD LSRII Flow Cytometer and analyzed by FlowJo 9.9.6.

\section{RNA-seq analysis}

RNA was isolated with Qiagen RNeasy Plus mini kit and submitted to the Yale Stem Cell Center for stranded library preparation. RNA-seq libraries were sequenced on an Illumina HiSeq4000 at 2x100 bp with the target of at least $2.5 \times 10^{7}$ reads per sample. For YUMMER1.7 data, reads were trimmed of 
Illumina adapters using Trimmomatic v0.39 ${ }^{15}$, and aligned to reference genome mm10 using STAR aligner v2.7.3a ${ }^{16}$ with parameters - winAnchorMultimapNmax 200 -outFilterMultimapNmax 100. The repeatmasker annotation file for $\mathrm{mm} 10$ was obtain using UCSC table browser ${ }^{17}$. Reads falling in annotated repeat regions were counted using featureCounts v1.6. $2^{18}$ and differential accessibility analysis was performed with DESeq2 v1.32 ${ }^{19}$. For 1445 data, reads were trimmed of Illumina adapters using Trimmomatic v0.39 and mapped to the fasta files for repeat elements using Salmon v0.99.0 20 . Fasta files for TE were downloaded from RepBase ${ }^{21}$.

\section{ChIP-seq analysis}

ChIP-seq libraries were sequenced on an Illumina HiSeq2000 instrument as 50 bp single-end reads. Reads were trimmed of adaptor sequences using Trimmomatic v0.39 ${ }^{15}$, and aligned to the reference genome mm10 using Bowtie2 ${ }^{22}$. Duplicates were marked using Picard Tools v2.9.0 (Broad Institute. version 2.9.0. "Picard Tools." Broad Institute, GitHub repository. http://broadinstitute.github.io/picard/) and reads were filtered using SAMTools v1.923. Peaks were called using MACS2 ${ }^{24}$ and bigwig files generated with deeptools v3.1. $3^{25}$ with parameter -normalizeUsing RPKM.

\section{Patient Dataset analysis}

The raw fastq files for gene expression profiling of pembrolizumab-treated patients were downloaded from Hugo et al ${ }^{3}$. ERV fasta was downloaded from Tokuyama et $\mathrm{al}^{26}$. Fastq files were aligned to a combined fasta file with ERV and gencode fasta sequences using Salmon v0.99.0 ${ }^{20}$. Raw read counts were normalized using variance stabilizing transformation (VST ${ }^{19}$. VST values were used for box plots and correlation. TCGA data were downloaded using cBioportal R package "cgdsr"27. Log2 RSEM values were used for plotting and correlations.

\section{Western blot analysis}

Whole cell lysates were prepared with high salt lysis buffer (50 mM Tris-HCl pH 7.9, $0.1 \mathrm{mM}$ EDTA pH 8.0, $320 \mathrm{mM} \mathrm{NaCl}, 0.5 \%$ NP40, 10\% Glycerol, $1 x$ Protease Inhibitor cocktail (11873580001, Roche)) and 1x phosphor stop (4906845001, Sigma-Aldrich). Histones were separated by centrifugation, resuspended in SDS loading buffer, and sonicated. Protein concentration of whole cell lysates was measured by Bradford assay (Bio-Rad) with known BSA standards. Samples in SDS loading buffer were heated for 10 minutes at $95^{\circ} \mathrm{C}$ and loaded onto 6,7 or $10 \%$ (whole cell lysates) or $15 \%$ (histones) SDS-PAGE gels. Membranes were blocked in 5\% non-fat milk or BSA in Tris-buffered saline $(50 \mathrm{mM}$ Tris- $\mathrm{HCl}, 138 \mathrm{mM} \mathrm{NaCl}, 2.7 \mathrm{mM}$ $\mathrm{KCl}, \mathrm{pH} 7.4$ ) with $0.05 \%$ Tween (TBS-T) and incubated with primary antibodies in the same buffer or $5 \%$ BSA in TBS-T overnight at $4^{\circ} \mathrm{C}$. Membranes were incubated with secondary anti-rabbit or anti-mouse antibodies for 1 hour at room temperature. Blots were visualized by KwikQuant Imager. Primary antibodies used included rabbit anti-KDM5B (HPA027179, Sigma), mouse anti-tubulin (T5168, Sigma), rabbit anti-histone H3 (ab1791, Abcam), rabbit anti-H3K4me3 (C42D8, CST), mouse anti-vinculin (V9131, Sigma), and rabbit anti-GAPDH (CST2118 14C10). 
Total RNA extraction, cDNA reverse transcription, and RT-qPCR were performed as previously described ${ }^{14}$ with primers listed in Extended Data Table 10.

\section{Immunoprecipitation}

Whole cell lysates were prepared with NP40 buffer (50mM Tris - $\mathrm{HCl} \mathrm{pH} \mathrm{7.4,} 150 \mathrm{mM} \mathrm{NaCl}, 1 \% \mathrm{NP}-40$ ) with 1x Protease Inhibitor cocktail (11873580001, Roche)) and 1x PhosSTOP (4906845001, Sigma-Aldrich) on ice for 30 minutes, with occasional vortexing. Cell lysates were centrifuged at 10,000xg for 15 mins at $4^{\circ} \mathrm{C}$. Pre-clearing supernatants with protein $A / G$ beads were done before incubating with anti-KDM5B antibody (NB100-97821, Novus biologicals) or anti-SETDB1 antibody (11231-1-AP, Proteintech) at $4^{\circ} \mathrm{C}$ overnight. Protein A/G beads were added and further incubated for 2 hours. Wash the beads with NP40 lysis buffer 5 times, add $25 \mathrm{ul}$ of $2 x$ SDS loading buffer and boil at $95-100^{\circ} \mathrm{C}$ for 5 minutes to denature the protein and separate from the beads. Endogenous Co-IP was preceded similarly with nuclear extracts prepared with NE-PER extraction reagents (Thermo Scientific 78833) and further diluted 4 times with IP buffer (50 mM Tris-HCl pH8.0, 0.01\% sodium deoxycholate, $150 \mathrm{mM} \mathrm{NaCl}, 1 \%$ Trixon X-100, 1xcomplete protease inhibitor and $1 \mathrm{x}$ PhosSTOP). Beads were washed with IP buffer.

\section{Chromatin Immunoprecipitation (ChIP)}

YUMMER1.7 WT, $k d m 5 b s g, k d m 5 b$ sg with GFP, $k d m 5 b$ sg with WT KDM5B, $k d m 5 b$ sg with enzymatic mutant KDM5B, $k d m 5 b$ sg with WT SETDB1 and 1445 cells in $15 \mathrm{~cm}$ plates were washed with PBS, then cross-linked with $19 \mathrm{ml}$ of fresh DMEM/F12+1\% formaldehyde for 10 minutes with gentle swirling at room temperature. $1 \mathrm{ml}$ of $2.5 \mathrm{M}$ glycine was added to achieve final concentration of $0.125 \mathrm{M}$ in $20 \mathrm{ml}$ and incubated at room temperature for another 5 minutes to quench the cross-link reaction. Cells were scraped in quenched medium into $50 \mathrm{ml}$ tube. P150 plate was washed with $20 \mathrm{ml}$ cold $1 \times$ PBS and remaining cells were scraped and transferred to $50 \mathrm{ml}$ tube. Cells were pelleted with $1000 \mathrm{xg}$ for 3 mins at $4^{\circ} \mathrm{C}$ and washed two more times with cold PBS on ice. $1 \times 10^{8}$ Pelleted cells were resuspended in $1 \mathrm{ml}$ sonication buffer (20mM Tris, pH8.0, $2 \mathrm{mM}$ EDTA, 0.5mM EGTA, $1 x$ protease inhibitors, $0.5 \%$ SDS and $0.5 \mathrm{mM}$ PMSF) and incubated on ice for 5-10 mins (To remove bubbles if needed, 1-2 $\mu$ l of PMSF can be added to top of the solution for each tube). Cells in sonication buffers can be flash frozen in liquid $\mathrm{N}_{2}$ and stored in $-80^{\circ} \mathrm{C}$ or preceded to sonication directly. Cells in sonication buffers were further diluted 3 times with sonication buffer to achieve final $3.3 \times 10^{4} \mathrm{cells} / \mu$ land sonicated with Qsonica Q800R2 with 70\% amplitude $15 \mathrm{~s}$ on, $45 \mathrm{~s}$ off for $35 \mathrm{~min}$ on total. After sonication, chromatin was centrifuged at max speed for 10 mins at $4^{\circ} \mathrm{C}$, and then supernatant was transferred. Aliquots to check fragment size were taken, added with elution buffer and $5 \mathrm{M} \mathrm{NaCl}$, and incubated at $65^{\circ} \mathrm{C}$ overnight (or>3.5h) in water bath. $20 \%$ input was saved at $-20^{\circ} \mathrm{C}$. Remaining chromatins were pre-cleared with protein $A / G$ beads. For each IP preclearing, $225 \mu$ l of diluted chromatin were incubated with $1 \mathrm{ml}$ IP buffer $(20 \mathrm{mM}$ Tris-HCl, pH8.0, $2 \mathrm{mM}$ EDTA, $0.5 \%$ Triton X-100, $150 \mathrm{mM} \mathrm{Nacl}$, and $10 \%$ glycerol) and $30 \mu \mathrm{l}$ of protein $\mathrm{A} / \mathrm{G}$ beads with rotation at $4^{\circ} \mathrm{C}$ for 1 hour. Pellet agarose beads, transfer cleared ChIP solution to a new tube and add $250 \mu \mathrm{l}$ more IP 
buffer to dilute SDS, then primary antibodies were added and incubated with rotation at $4^{\circ} \mathrm{C}$ overnight. Anti-H3K4me3 (ab8580, Abcam), anti-H3K9me3 (ab8898, Abcam), anti-KDM5B antibody (NB100-97821, Novus biologicals), anti-SETDB1 antibody (11231-1-AP, Proteintech) or Normal Rabbit IgG control (\#2729, CST) were used. Next day, protein A/G beads were washed three time with IP buffer. After wash, $30 \mu \mathrm{l}$ of protein $\mathrm{A} / \mathrm{G}$ slurry were added to each IP reaction and incubated with rotation at $4^{\circ} \mathrm{C}$ for $2 \mathrm{~h}$. After rotation, the beads were washed 1 time with low salt wash buffer $(10 \mathrm{mM}$ Tris-HCl pH8.0, 2 mM EDTA, 1\% Triton X$100,150 \mathrm{mM} \mathrm{NaCl}, 0.1 \%$ SDS), 3 times with high salt wash buffer(10mM Tris-HCl pH8.0, 2 mM EDTA, 1\% Triton X-100, $500 \mathrm{mM} \mathrm{NaCl}, 0.1 \%$ SDS), 1 time LiCl wash (10mM Tris-HCl pH8.0, 2 mM EDTA, $250 \mathrm{mM}$ $\mathrm{LiCl}, 1 \% \mathrm{NP} 40$, and $1 \%$ sodium deoxycholate) and 2 times with TE buffer wash. DNA was eluted elution buffer $100 \mathrm{mM} \mathrm{NaHCO}_{3}, 1 \% \mathrm{SDS}$ ) and shaked at $65^{\circ} \mathrm{C}$ at $1400 \mathrm{rpm}$ for $20 \mathrm{~min} .5 \mathrm{M} \mathrm{NaCl}$ was added to the elution and incubated at $65^{\circ} \mathrm{C}$ overnight. $1 \mathrm{M}$ Tris- $\mathrm{HCl}$ pH8.0, glycogen, and proteinase $\mathrm{K}$ were added and incubated at $50^{\circ} \mathrm{C}$ for $1 \mathrm{~h}$ to reverse crosslinking. DNA was extracted with phenol:chloroform:isoamyl alcohol (25:24:1 v/v) 3 times, precipitated with ethanol, washed, air dried and resuspended in $100 \mu \mathrm{H}_{2} \mathrm{O}$. ChIP-qPCR analysis was performed using primers described in Extended Data Table 10.

\section{Tissue Microarray Production, Staining, and Tissue Cytometry Analysis of the Patient Cohort}

We used 65 melanoma tissue samples from patients treated at our institution with PD-1 inhibitors with or without anti-CTLA-4 between 2009 and 2015. Patient tumor characteristics and clinical information for the sixty-five patients have been previously described ${ }^{28}$. All patient tissue samples were collected with the approval from Yale Human Investigations Committee under protocol \# 0609001869. The specimens were obtained from formalin-fixed, paraffin embedded (FFPE) patient melanoma tissues; a pathologist examined FFPE tissue sections from each case and selected representative regions of invasive tumor for coring; three punches from each specimen were obtained and embedded into a master tissue microarray (TMA) block using methods previously described ${ }^{28,29}$. FFPE cell line pellets were used for antibody staining controls. TMA slides were processed as previously described ${ }^{28,29}$. Antibodies included antiKDM5B antibody (sigma HPA027179), anti-S100 (BioGenex, clone 15E2E2, MU058-UC) and anti-HMB45 (BioGenex, MU001A-UC). Briefly, TMA slides were deparaffinized and hydrated followed by antigen retrieval. Slides were boiled for 20 minutes in a pressure cooker containing $6.5 \mathrm{mM}$ sodium citrate $(\mathrm{pH}$ 6.0). Endogenous peroxidase activity was blocked using hydrogen peroxide solution. Unspecific staining was blocked in $0.3 \%$ bovine serum albumin solution before slides were concurrently stained with a cocktail of mouse anti-S100 and mouse anti-HMB45 to detect tumor cells and anti-KDM5B antibody. For visualization of $\mathrm{S} 100$ and $\mathrm{HMB} 45$ staining we used the goat anti-mouse $\lg \mathrm{G}$ conjugated to Alexa 546 (ThermoFisher Scientific, A-11030) in anti-rabbit amplification reagent (Envision; Dako, K4003). KDM5B staining was visualized with Cyanine-5-tyramide (Perkin Elmer, SAT705A001EA). Nuclei were visualized by incubating the slides with 4,6-diamidine-2-phenylindole (DAPI). Coverslips were mounted with ProLong Gold antifade medium (Invitrogen/Life Technologies, P36931).

The tissue microarray was imaged and analyzed by tissue cytometry according to previously published method $^{30}$. Briefly, images were acquired on the TissueFAXS Quantitative Imaging System 
(TissueGnostics, Vienna, Austria). The entire slide $\left(75 \times 25 \mathrm{~mm}^{2}\right)$ was scanned at low magnification using a $5 \times$ objective. The low magnification scan was used to identify the location of the tissue on the slide. A region gate was drawn around each TMA for acquisition at $20 \times$ high magnification ${ }^{30}$. The resulting images were exported as full resolution TIFF files in greyscale for each dye channel. Image processing and analysis was performed using StrataQuest software version 6.0.1.145 (TissueGnostics, Vienna, Austria). Image processing included isolation of each TMA spot and creation of pseudocolored multiplexed images from the greyscale images for each TMA spot ${ }^{30}$. Algorithms were created to isolate each cell in the multiplexed image and identify the positive cells. Global standard measurements were computed for mean grey level intensity (MGLI). Histograms and 2D dot scatterplots were created to enumerate the number of positive and negative cells for each biomarker ${ }^{30}$. Threshold cutoffs depicted by horizontal and/or vertical lines were determined manually by backgating to the greyscale and multiplexed tissue images for each biomarker. Using the backgating algorithm, threshold cutoffs were manually positioned to include or exclude cells and/or cell clusters ${ }^{30}$.

\section{Declarations}

Acknowledgements: We thank members of the Yan, Bosenberg, and Iwasaki laboratories for their helpful discussion and support. We thank Dr. Jeffrey J. Ishizuka for helpful discussions. We thank Dr. Ruth Halaban from the Department of Dermatology at Yale University for providing the human melanoma cell lines used in this study. We thank Dr. Frank J. Rauscher, III from Wistar Institute for providing pCMV.FlagSETDB1 (WT and dead mutant) plasmids. Sequencing done at Yale Stem Cell Center Genomics Core facility was supported by the Connecticut Regenerative Medicine Research Fund and the Li Ka Shing Foundation. We thank Yale Pathology Tissue Services and Comparative Pathology Research Core for histology and immunohistochemistry service.

These studies were supported in part by grants from the NIH Yale SPORE in Skin Cancer, NCI P50 CA121974 (to M.W.B., H.M.K., M.S. and Q.Y.), R01 CA237586 (to Q.Y.), R01 CA227473 and R01 CA216846 (to H.M.K.), Department of Defense W81XWH-13-1-0235 (to Q.Y.) and W81XWH-16-1-0306 and W81XWH20-1-0360 (to S-M.Z.), a pilot grant from Yale Cancer Center (M.W.B., Q.Y. and A.I.), the Melanoma Research Foundation (to M.W.B. and Q.Y.), the Lung Cancer Research Foundation-LUNGevity and Melanoma Research Alliance Award \#308721 (to L.B.J.), and Melanoma Research Alliance Young Investigator Award (to K.R.M.B.).

Author contributions: S-M.Z., M.W.B., and Q.Y. conceived and designed the study; S-M.Z. did the most experiments with helps from X.L. and W.L.C.; W.L.C. performed the retroelement analysis of RNA-seq and ChIP-seq; D.T. and M.K.M. analyzed RNA-seq; G.M. performed some TCGA bioinformatic analysis. C.J.B. performed histology analysis and captured IHC images; K.R.M.B performed capturing/quantification/analytical analysis for TMA; L.B.J. performed TMA staining; M.S. and H.M.K. provided clinical patient samples; W.B., and A.I. provided input on study design and data analysis. S-M.Z., M.W.B., and Q.Y. wrote the manuscript. 


\section{References}

1 Kearney, C. J. et al. Tumor immune evasion arises through loss of TNF sensitivity. Sci Immuno/3, doi:10.1126/sciimmunol.aar3451 (2018).

2 Trujillo, J. A., Sweis, R. F., Bao, R. \& Luke, J. J. T Cell-Inflamed versus Non-T Cell-Inflamed Tumors: A Conceptual Framework for Cancer Immunotherapy Drug Development and Combination Therapy Selection. Cancer Immunol Res 6, 990-1000, doi:10.1158/2326-6066.CIR-18-0277 (2018).

3 Hugo, W. et al. Genomic and Transcriptomic Features of Response to Anti-PD-1 Therapy in Metastatic Melanoma. Cell 165, 35-44, doi:10.1016/j.cell.2016.02.065 (2016).

$4 \quad$ Wang, J. et al. UV-induced somatic mutations elicit a functional T cell response in the YUMMER1.7 mouse melanoma model. Pigment Cell Melanoma Res 30, 428-435, doi:10.1111/pcmr.12591 (2017).

5 Sharma, P., Hu-Lieskovan, S., Wargo, J. A. \& Ribas, A. Primary, Adaptive, and Acquired Resistance to Cancer Immunotherapy. Cell 168, 707-723, doi:10.1016/j.cell.2017.01.017 (2017).

6 Held, M. A. et al. Characterization of melanoma cells capable of propagating tumors from a single cell. Cancer Res 70, 388-397, doi:10.1158/0008-5472.CAN-09-2153 (2010).

7 Baum, A., Sachidanandam, R. \& Garcia-Sastre, A. Preference of RIG-I for short viral RNA molecules in infected cells revealed by next-generation sequencing. Proc Natl Acad Sci U S A 107, 16303-16308, doi:10.1073/pnas.1005077107 (2010).

$8 \mathrm{Mu}, \mathrm{X} ., \mathrm{Ahmad}, \mathrm{S}$. \& Hur, S. Endogenous Retroelements and the Host Innate Immune Sensors. Adv Immuno/ 132, 47-69, doi:10.1016/bs.ai.2016.07.001 (2016).

9 Kato, M., Takemoto, K. \& Shinkai, Y. A somatic role for the histone methyltransferase Setdb1 in endogenous retrovirus silencing. Nat Commun 9, 1683, doi:10.1038/s41467-018-04132-9 (2018).

$10 \mathrm{Xu}$, J. \& Kidder, B. L. H4K20me3 co-localizes with activating histone modifications at transcriptionally dynamic regions in embryonic stem cells. BMC Genomics 19,514 , doi:10.1186/s12864018-4886-4 (2018).

11 Sheng, W. et al. LSD1 Ablation Stimulates Anti-tumor Immunity and Enables Checkpoint Blockade. Cell 174, 549-563 e519, doi:10.1016/j.cell.2018.05.052 (2018).

12 Chiappinelli, K. B. et al. Inhibiting DNA Methylation Causes an Interferon Response in Cancer via dsRNA Including Endogenous Retroviruses. Cel/ 162, 974-986, doi:10.1016/j.cell.2015.07.011 (2015).

13 Roulois, D. et al. DNA-Demethylating Agents Target Colorectal Cancer Cells by Inducing Viral Mimicry by Endogenous Transcripts. Cell 162, 961-973, doi:10.1016/j.cell.2015.07.056 (2015). 
14 Liu, X. et al. KDM5B Promotes Drug Resistance by Regulating Melanoma-Propagating Cell Subpopulations. Mol Cancer Ther 18, 706-717, doi:10.1158/1535-7163.MCT-18-0395 (2019).

15 Bolger, A. M., Lohse, M. \& Usadel, B. Trimmomatic: a flexible trimmer for Illumina sequence data. Bioinformatics 30, 2114-2120, doi:10.1093/bioinformatics/btu170 (2014).

16 Dobin, A. et al. STAR: ultrafast universal RNA-seq aligner. Bioinformatics 29, 15-21, doi:10.1093/bioinformatics/bts635 (2013).

17 Karolchik, D. et al. The UCSC Table Browser data retrieval tool. Nucleic Acids Res 32, D493-496, doi:10.1093/nar/gkh103 (2004).

18 Liao, Y., Smyth, G. K. \& Shi, W. featureCounts: an efficient general purpose program for assigning sequence reads to genomic features. Bioinformatics 30, 923-930, doi:10.1093/bioinformatics/btt656 (2014).

19 Love, M. I., Huber, W. \& Anders, S. Moderated estimation of fold change and dispersion for RNAseq data with DESeq2. Genome Biol 15, 550, doi:10.1186/s13059-014-0550-8 (2014).

20 Patro, R., Duggal, G., Love, M. I., Irizarry, R. A. \& Kingsford, C. Salmon provides fast and bias-aware quantification of transcript expression. Nat Methods 14, 417-419, doi:10.1038/nmeth.4197 (2017).

21 Bao, W., Kojima, K. K. \& Kohany, O. Repbase Update, a database of repetitive elements in eukaryotic genomes. Mob DNA 6, 11, doi:10.1186/s13100-015-0041-9 (2015).

22 Langmead, B. \& Salzberg, S. L. Fast gapped-read alignment with Bowtie 2. Nat Methods 9, 357359, doi:10.1038/nmeth.1923 (2012).

$23 \mathrm{Li}, \mathrm{H}$. et al. The Sequence Alignment/Map format and SAMtools. Bioinformatics 25, 2078-2079, doi:10.1093/bioinformatics/btp352 (2009).

24 Zhang, Y. et al. Model-based analysis of ChIP-Seq (MACS). Genome Bio/ 9, R137, doi:10.1186/gb2008-9-9-r137 (2008).

25 Ramirez, F. et al. deepTools2: a next generation web server for deep-sequencing data analysis. Nucleic Acids Res 44, W160-165, doi:10.1093/nar/gkw257 (2016).

26 Tokuyama, M. et al. ERVmap analysis reveals genome-wide transcription of human endogenous retroviruses. Proc Natl Acad Sci U S A 115, 12565-12572, doi:10.1073/pnas.1814589115 (2018).

27 Cerami, E. et al. The cBio cancer genomics portal: an open platform for exploring multidimensional cancer genomics data. Cancer Discov 2, 401-404, doi:10.1158/2159-8290.CD-12-0095 (2012). 
28 Kluger, H. M. et al. PD-L1 Studies Across Tumor Types, Its Differential Expression and Predictive Value in Patients Treated with Immune Checkpoint Inhibitors. Clin Cancer Res 23, 4270-4279, doi:10.1158/1078-0432.CCR-16-3146 (2017).

29 Kluger, H. M. et al. Characterization of PD-L1 Expression and Associated T-cell Infiltrates in Metastatic Melanoma Samples from Variable Anatomic Sites. Clin Cancer Res 21, 3052-3060, doi:10.1158/1078-0432.CCR-14-3073 (2015).

30 Blenman, K. R. M. \& Bosenberg, M. W. Immune Cell and Cell Cluster Phenotyping, Quantitation, and Visualization Using In Silico Multiplexed Images and Tissue Cytometry. Cytometry A 95, 399-410, doi:10.1002/cyto.a.23668 (2019).

\section{Figures}


Figure 1.

a

\begin{tabular}{|llr|}
\hline \multicolumn{3}{|c|}{$\begin{array}{c}\text { Top } 5 \text { gene ontology (GO) categories negatively } \\
\text { correlated with }\end{array}$ KDM5B RNA levels (TCGA melanoma data) } \\
\hline GO Term & \multicolumn{1}{c|}{ Description } & \multicolumn{1}{c|}{ FDR } \\
\hline GO:0002376 & immune system process & $2.61 \mathrm{E}-17$ \\
GO:0002682 & regulation of immune system process & $8.01 \mathrm{E}-15$ \\
GO:0050776 & regulation of immune process & $8.25 \mathrm{E}-14$ \\
GO:0002684 & positive regulation of immune system & $2.08 \mathrm{E}-13$ \\
GO:0019221 & process & cytokine-mediated signaling pathways 4.07E-12 \\
\hline
\end{tabular}

C

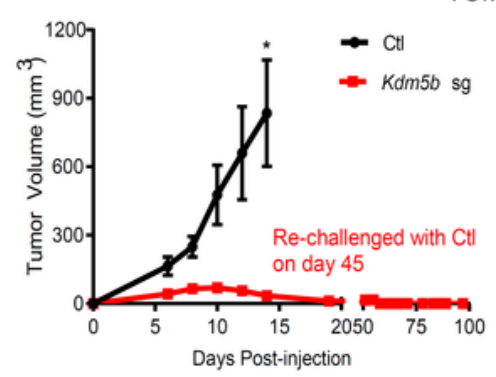

b

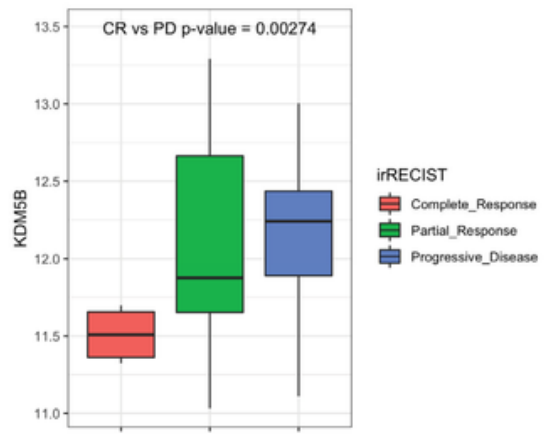

d



YUMMER1.7

e
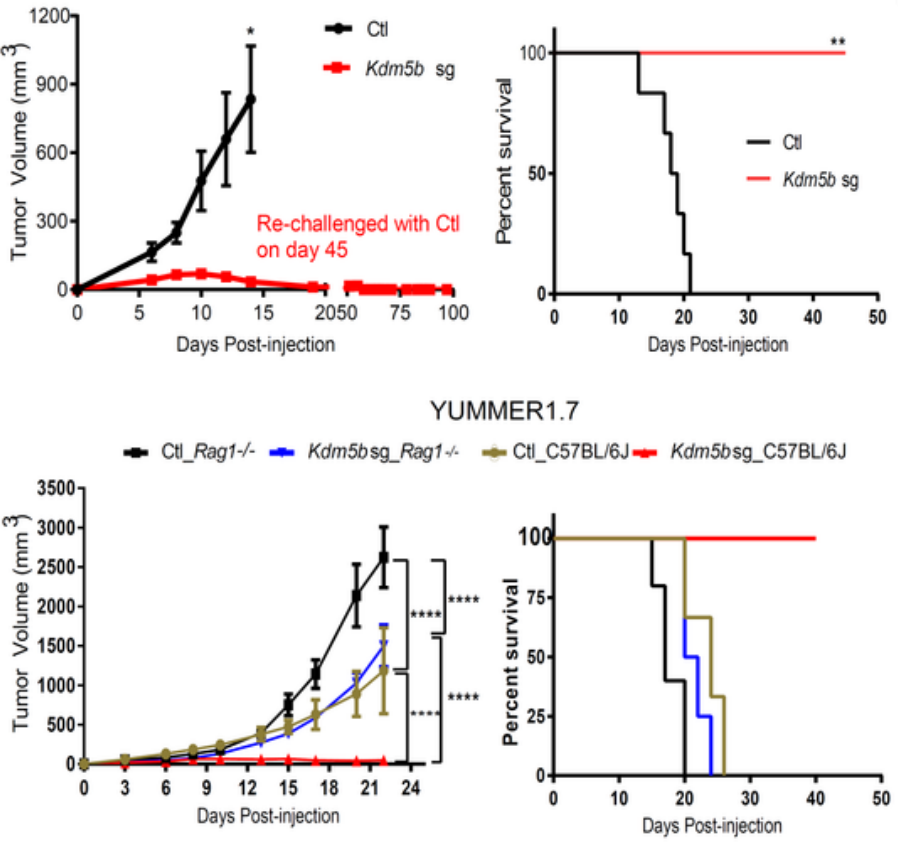

f

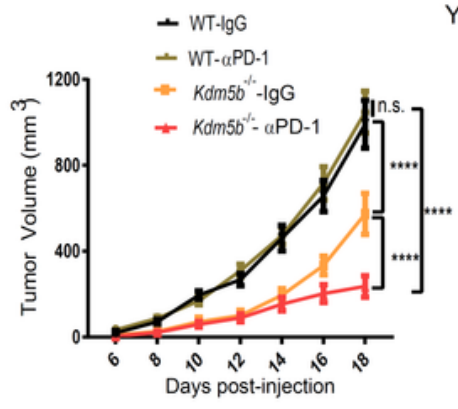

YUMM1.7

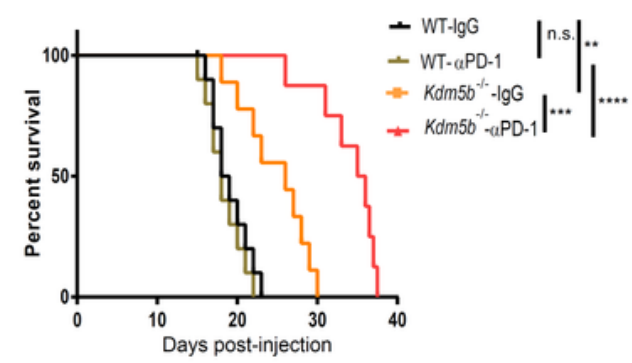

Figure 1

Deletion of KDM5B induces anti-tumor immunity, in an adaptive immune system dependent manner. a, Top 5 gene ontology pathways identified from Gorilla Gene Ontology (GO) analysis of 9,336 genes negatively correlated with KDM5B from The Cancer Genome Atlas (TCGA) melanoma dataset. FDR, false discovery rate. b, Box plot (center line: median, box limits: upper and lower quartiles, whiskers: 1.5x interquartile range, points outside whiskers: outliers) showing KDM5B mRNA levels from RNA-seq 
analysis of tumors sampled prior to anti-PD-1 therapy from patients with complete response (CR), partial response, or progressive disease (PD) from Hugo et al3. Statistical significance was computed with unpaired two-sided Student's t test. c, Tumor growth curves (left) and survival curves (right) of C57BL/6 mice injected with control (black) or Kdm5b sgRNA (red) $(n=6)$ YUMMER1.7 cells. Mice injected with Kdm5b sgRNA cells had completely regressed tumors, and were re-challenged with control tumor cells on day 45 after initial tumor injection. Data shown are representative results from two independent experiments. Unpaired two-sided Student's t-test ${ }^{*} p<0.05(p=0.032)$ for tumor growth curve at day 14; Data are represented as mean \pm SEM; log-rank test ${ }^{*} p<0.01(p=0.004)$ for survival curve. $d$, Flow cytometry analysis of the percentages of CD4+ and CD8+ cells in total CD45+ immune cells among control and Kdm5b sgRNA tumors. Unpaired two-sided Student's t-test for each comparison, ${ }^{*} \mathrm{p}<0.05$ $(p=0.036)$ for $C D 4+\%$; ${ }^{*}<0.05(p=0.024)$ for $C D 8+\%$. Data are represented as mean \pm SD. e, Tumor growth curves (left) and survival curves (right) of wild type C57BL/6J or Rag1-/- injected with control or Kdm5b sgRNA YUMMER1.7 cells $(n=5)$. Data are represented as mean \pm SEM. 2 way ANOVA analysis for comparison at day $22,{ }^{* \star \star *} p<0.0001$. log-rank test were used for survival curves: ${ }^{*} p<0.05(p=0.014)$ for Ctl_C57BL/6J vs Kdm5b sg_C57BL/6J; * ${ }^{*}<0.05$ ( $\left.p=0.042\right)$ for Ctl_Rag1-/-vs Ctl_C57BL/6J ; * $<<0.05$ $(\mathrm{p}=0.038)$ for Ctl_Rag1-/-vs Kdm5b sg_Rag1-/, ** $p<0.01$ ( $\mathrm{p}=0.006)$ for Kdm5b sg_C57BL/6J vs Kdm5b sg_Rag1-/; n.s. for CtI_C57BL/6J vs Kdm5b sg_Rag1-/, **p<0.01 ( $p=0.006)$ for CtI_Rag1-/-vs Kdm5b sg _ C57BL/6J. f, Tumor growth curves and survival curves of C57BL/6 mice injected with YUMM1.7 or YUMM1.7-Kdm5b-/- cells treated with IgG control or anti-PD-1 antibody ( $\mathrm{n}=9$ or10). Data are represented as mean \pm SEM . 2-way ANOVA analysis for comparison at day $18, \star \star \star \star p<0.0001$. log-rank tests were used for survival curves: ${ }^{\star \star} p<0.01(\mathrm{p}=0.002)$ for WT_IgG vs Kdm5b sg_IgG; $* \star \star \star p<0.0001$ for WT_aPD-1 vs kdm5b sg_aPD-1; n.s. for WT_IgG vs WT_aPD-1; *** $<<0.001$ ( $p=0.0003)$ for Kdm5b sg_lgG vs Kdm5b sg_ aPD-1. 
Figure 1.

a

\begin{tabular}{|llr|}
\hline \multicolumn{3}{|c|}{$\begin{array}{c}\text { Top } 5 \text { gene ontology (GO) categories negatively } \\
\text { correlated with }\end{array}$ KDM5B RNA levels (TCGA melanoma data) } \\
\hline GO Term & \multicolumn{1}{c|}{ Description } & \multicolumn{1}{c|}{ FDR } \\
\hline GO:0002376 & immune system process & $2.61 \mathrm{E}-17$ \\
GO:0002682 & regulation of immune system process & $8.01 \mathrm{E}-15$ \\
GO:0050776 & regulation of immune process & $8.25 \mathrm{E}-14$ \\
GO:0002684 & positive regulation of immune system & $2.08 \mathrm{E}-13$ \\
GO:0019221 & process & cytokine-mediated signaling pathways 4.07E-12 \\
\hline
\end{tabular}

C

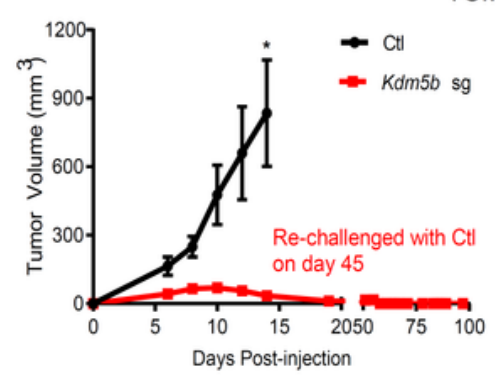

b

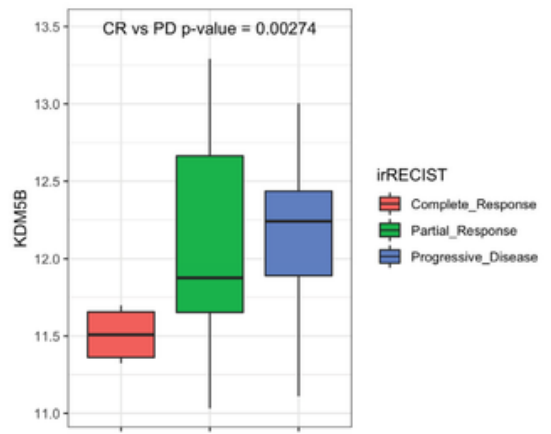

d

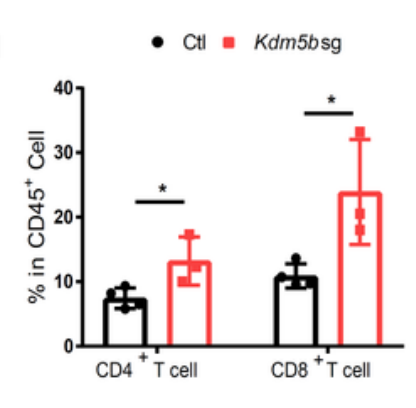

YUMMER1.7

e
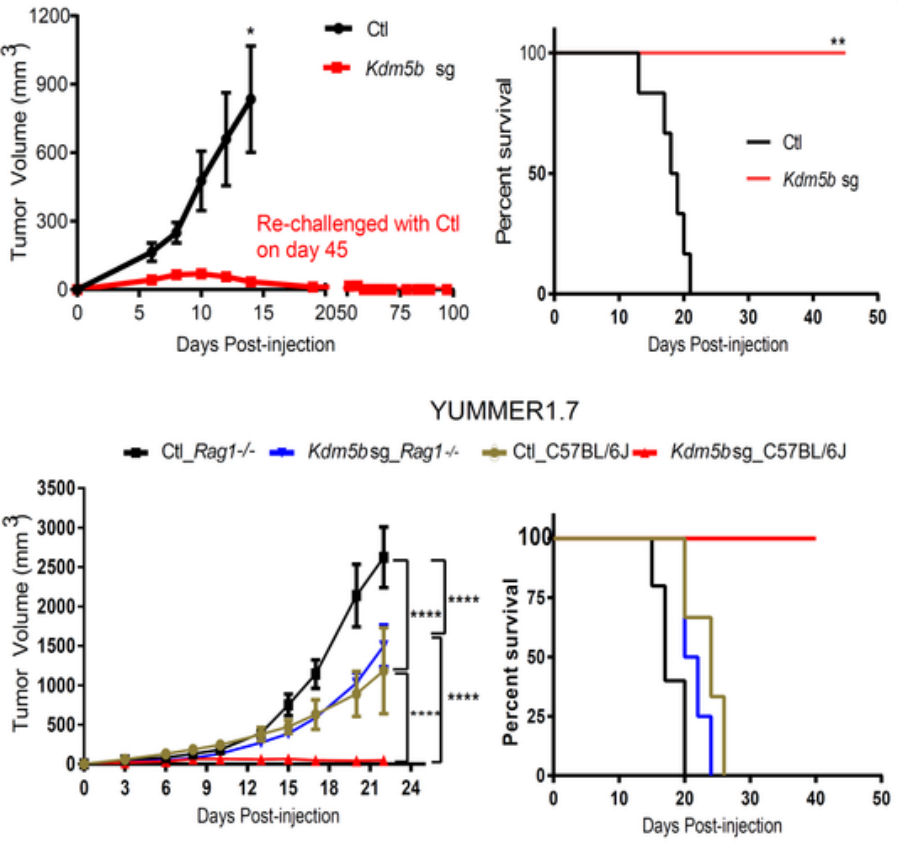

f

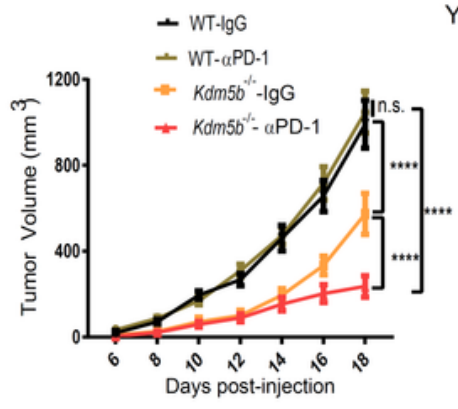

YUMM1.7

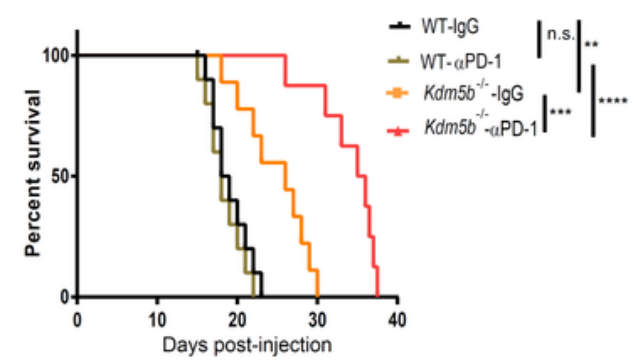

Figure 1

Deletion of KDM5B induces anti-tumor immunity, in an adaptive immune system dependent manner. a, Top 5 gene ontology pathways identified from Gorilla Gene Ontology (GO) analysis of 9,336 genes negatively correlated with KDM5B from The Cancer Genome Atlas (TCGA) melanoma dataset. FDR, false discovery rate. b, Box plot (center line: median, box limits: upper and lower quartiles, whiskers: 1.5x interquartile range, points outside whiskers: outliers) showing KDM5B mRNA levels from RNA-seq 
analysis of tumors sampled prior to anti-PD-1 therapy from patients with complete response (CR), partial response, or progressive disease (PD) from Hugo et al3. Statistical significance was computed with unpaired two-sided Student's t test. c, Tumor growth curves (left) and survival curves (right) of C57BL/6 mice injected with control (black) or Kdm5b sgRNA (red) $(n=6)$ YUMMER1.7 cells. Mice injected with Kdm5b sgRNA cells had completely regressed tumors, and were re-challenged with control tumor cells on day 45 after initial tumor injection. Data shown are representative results from two independent experiments. Unpaired two-sided Student's t-test ${ }^{*} p<0.05(p=0.032)$ for tumor growth curve at day 14; Data are represented as mean \pm SEM; log-rank test ${ }^{*} p<0.01(p=0.004)$ for survival curve. $d$, Flow cytometry analysis of the percentages of CD4+ and CD8+ cells in total CD45+ immune cells among control and Kdm5b sgRNA tumors. Unpaired two-sided Student's t-test for each comparison, ${ }^{*} \mathrm{p}<0.05$ $(p=0.036)$ for $C D 4+\%$; ${ }^{*}<0.05(p=0.024)$ for $C D 8+\%$. Data are represented as mean \pm SD. e, Tumor growth curves (left) and survival curves (right) of wild type C57BL/6J or Rag1-/- injected with control or Kdm5b sgRNA YUMMER1.7 cells $(n=5)$. Data are represented as mean \pm SEM. 2 way ANOVA analysis for comparison at day $22,{ }^{* \star \star *} p<0.0001$. log-rank test were used for survival curves: ${ }^{*} p<0.05(p=0.014)$ for Ctl_C57BL/6J vs Kdm5b sg_C57BL/6J; * ${ }^{*}<0.05$ ( $\left.p=0.042\right)$ for Ctl_Rag1-/-vs Ctl_C57BL/6J ; * $<<0.05$ $(\mathrm{p}=0.038)$ for Ctl_Rag1-/-vs Kdm5b sg_Rag1-/, ** $p<0.01$ ( $\mathrm{p}=0.006)$ for Kdm5b sg_C57BL/6J vs Kdm5b sg_Rag1-/; n.s. for CtI_C57BL/6J vs Kdm5b sg_Rag1-/, **p<0.01 ( $p=0.006)$ for CtI_Rag1-/-vs Kdm5b sg _ C57BL/6J. f, Tumor growth curves and survival curves of C57BL/6 mice injected with YUMM1.7 or YUMM1.7-Kdm5b-/- cells treated with IgG control or anti-PD-1 antibody ( $\mathrm{n}=9$ or10). Data are represented as mean \pm SEM . 2-way ANOVA analysis for comparison at day $18, \star \star \star \star p<0.0001$. log-rank tests were used for survival curves: ${ }^{\star \star} p<0.01(\mathrm{p}=0.002)$ for WT_IgG vs Kdm5b sg_IgG; $* \star \star \star p<0.0001$ for WT_aPD-1 vs kdm5b sg_aPD-1; n.s. for WT_IgG vs WT_aPD-1; *** $<<0.001$ ( $p=0.0003)$ for Kdm5b sg_lgG vs Kdm5b sg_ aPD-1. 
Figure 2.

a
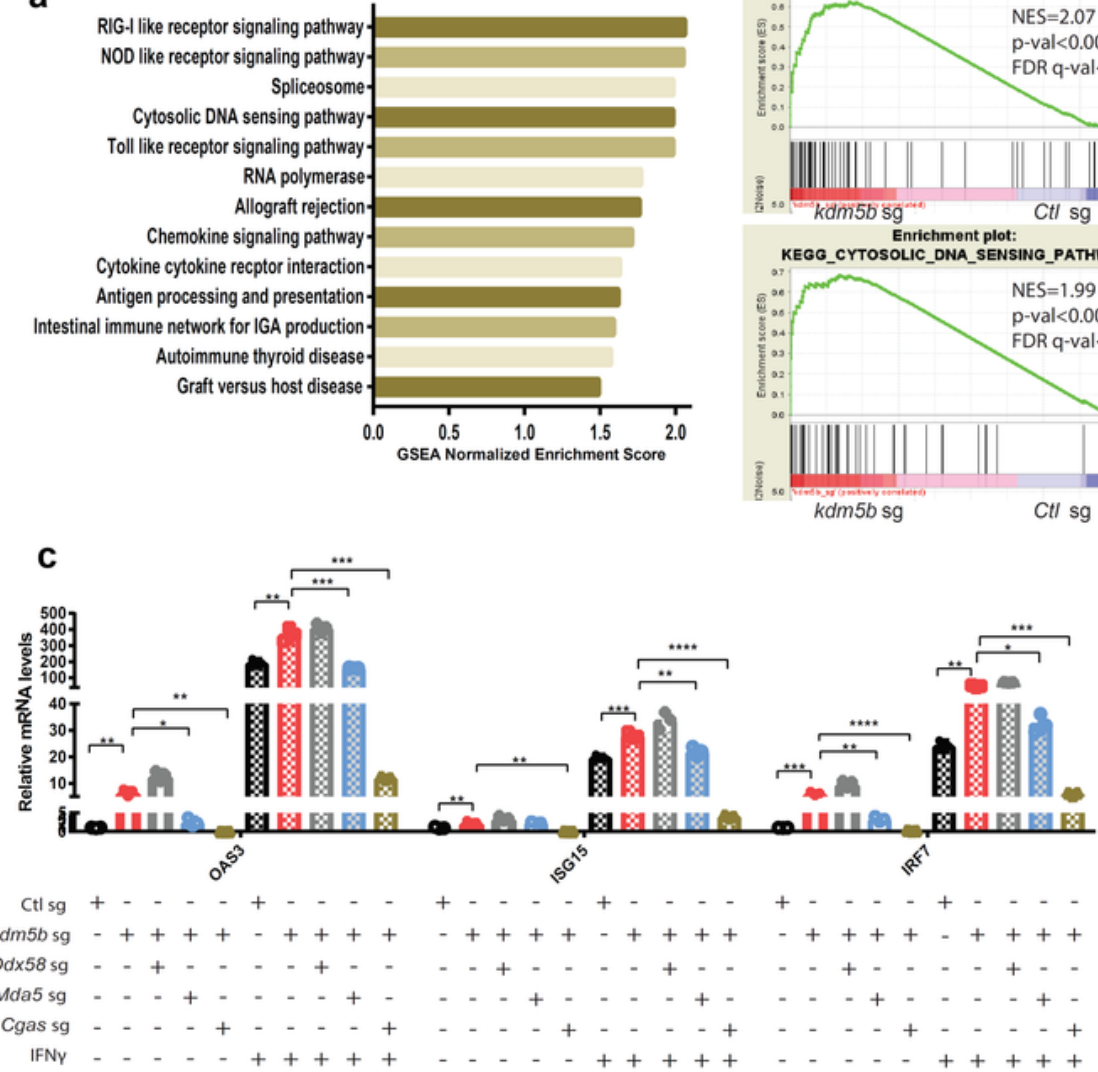

d
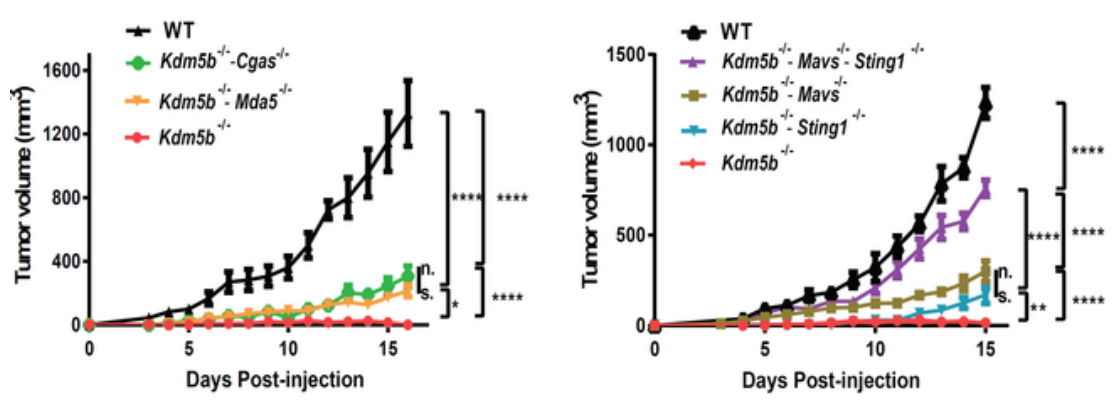
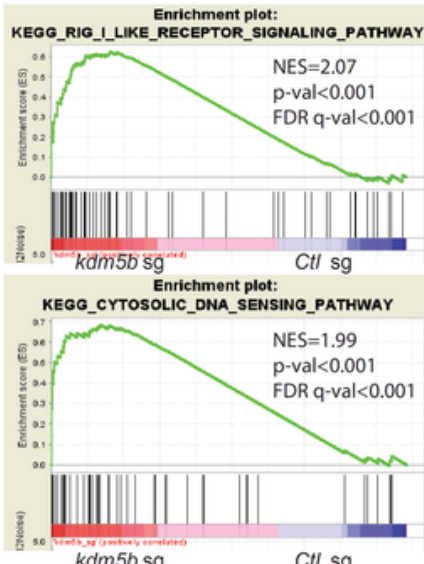

sg
Days Post-injection
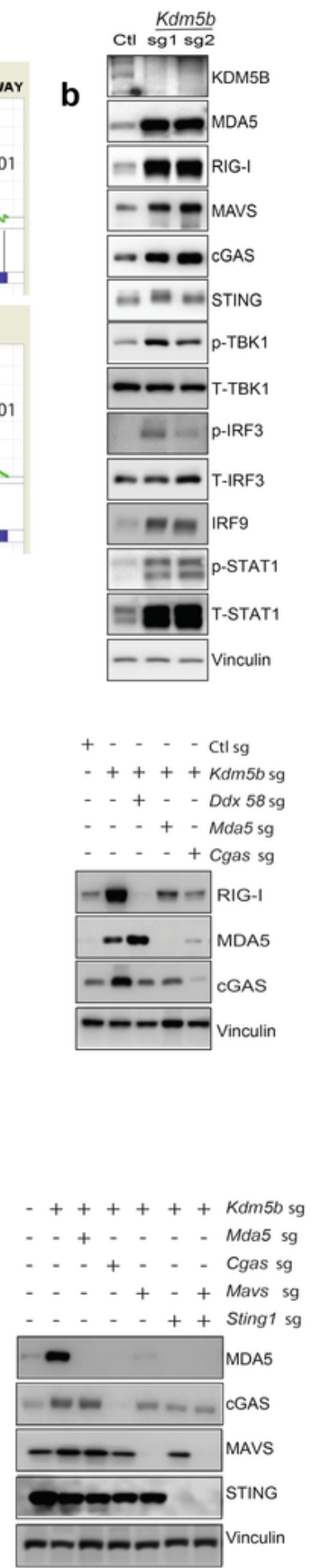

Figure 2

Deletion of KDM5B activates the type I interferon response through both cytosolic RNA and DNA sensing pathways. a, GSEA comparing RNA-seq data from Kdm5b sg YUMMER1.7 cells to control YUMMER1.7 cells. Shown on the left is the top upregulated pathways (FDR<0.05). Shown on the right are GSEA plots from two upregulated pathways selected from the left panel. b, Western blot analyses of DNA and RNA sensors, as well as signaling proteins involved in the type I IFN pathway. T-, total; p-, phosphorylated. c, RT- 
qPCR analysis of selected ISGs in the indicated cell lines, treated with or without of $10 \mathrm{ng}$ IFNy for $24 \mathrm{~h}$. Data are represented as mean \pm SD. Unpaired two-sided Students' $t$ tests. ${ }^{*} p<0.05 ;{ }^{* \star} p<0.01 ;{ }^{* \star \star} p<0.001$; $\star \star \star \star * p<0.0001$. Western blot analyses of these cell lines are shown on the right. $d$, Tumor growth curves of C57BL/ 6 mice injected with YUMMER1.7 cells of the indicated genotypes. Data are represented as mean \pm SEM. 2-way ANOVA analysis for comparison at day 16 (left) or 15 (middle) $n=6,{ }^{*} p<0.05$; ${ }^{\star \star} p<0.01$, $\star * \star * p<0.0001$. Western blot analyses of these cell lines are shown on the right.

Figure 2.

a

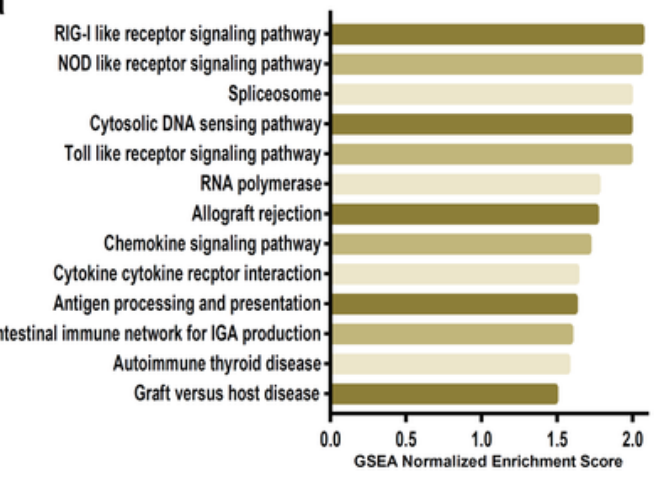

C
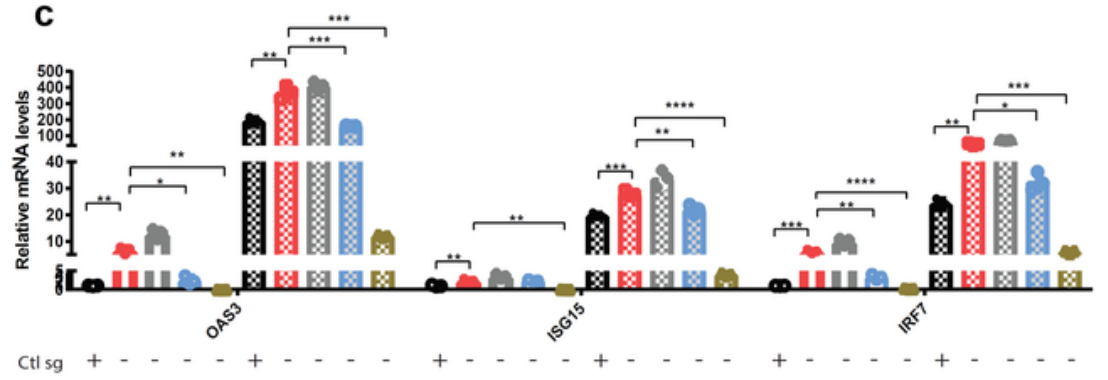

dmsbsg -++++-++++-++++-++++-++++-++++

$\int d \times 58 \mathrm{sg}-\ldots+\ldots+\cdots+\cdots+\cdots+\cdots+\cdots$

Uda5sg - - + - - + - - + - - + + - + - - +

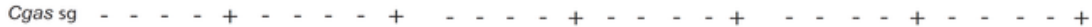

IFNY $\ldots \ldots+++++\ldots++++\ldots++++$

d
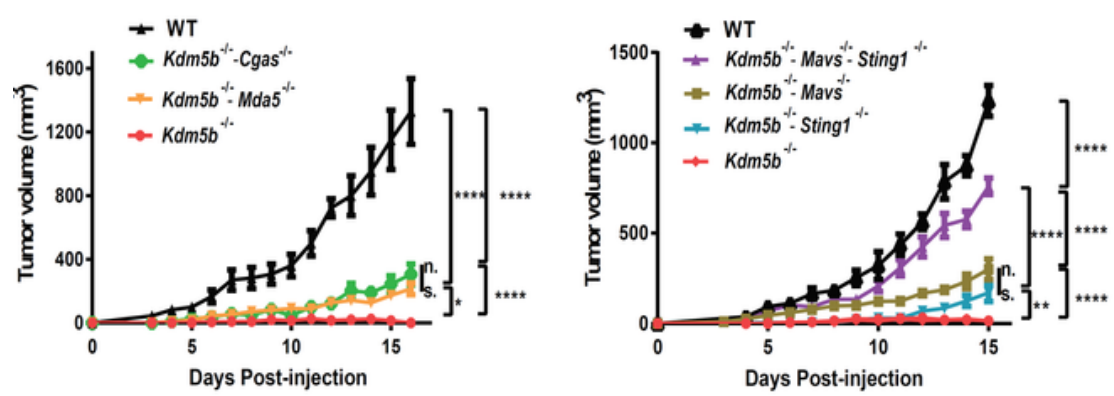

b
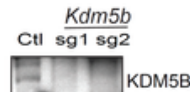

MDA5

- RIG-I

-0 mavs

$-\boldsymbol{\omega}$ cGAS

$\omega=-$ STING

- - p-TBK1

- - T-TBK1

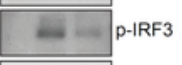

$-0-$ T-IRF3

$=$ IRF9

$E \equiv$ p-STAT 1

T-STAT1

$-\cdots$ Vinculin

$+\cdots-\mathrm{Ctlsg}^{-\cdots}$

+++ Kdm5bsg

$-\cdots+\cdots$

$-\cdots+-M d a 5 s g$

- . - + Cgas sg

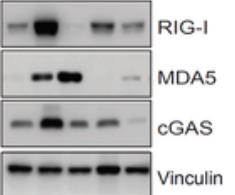

$-++++++K d m 5 b s g$

- - + - - - Mda5 sg

- - + + - - Cgas sg

- - - + + + Mavs sg

- - - - + + Sting1 sg

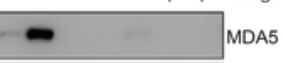

$=-\quad-\ldots$ CGAS

- - - - MAVS

STING

ローーーーーVinculin 
Deletion of KDM5B activates the type I interferon response through both cytosolic RNA and DNA sensing pathways. a, GSEA comparing RNA-seq data from Kdm5b sg YUMMER1.7 cells to control YUMMER1.7 cells. Shown on the left is the top upregulated pathways $(F D R<0.05)$. Shown on the right are GSEA plots from two upregulated pathways selected from the left panel. $b$, Western blot analyses of DNA and RNA sensors, as well as signaling proteins involved in the type I IFN pathway. T-, total; $\mathrm{p}$-, phosphorylated. c, RTqPCR analysis of selected ISGs in the indicated cell lines, treated with or without of $10 \mathrm{ng}$ IFNy for $24 \mathrm{~h}$. Data are represented as mean \pm SD. Unpaired two-sided Students' $t$ tests. ${ }^{*} p<0.05 ;{ }^{\star \star} p<0.01 ;{ }^{* \star \star} p<0.001$; $\star \star \star \star x<0.0001$. Western blot analyses of these cell lines are shown on the right. $d$, Tumor growth curves of C57BL/ 6 mice injected with YUMMER1.7 cells of the indicated genotypes. Data are represented as mean \pm SEM. 2-way ANOVA analysis for comparison at day 16 (left) or 15 (middle) $n=6,{ }^{\star} p<0.05$; ${ }^{\star \star} p<0.01$, $\star \star \star \star x<0.0001$. Western blot analyses of these cell lines are shown on the right. 
Figure 3.

a

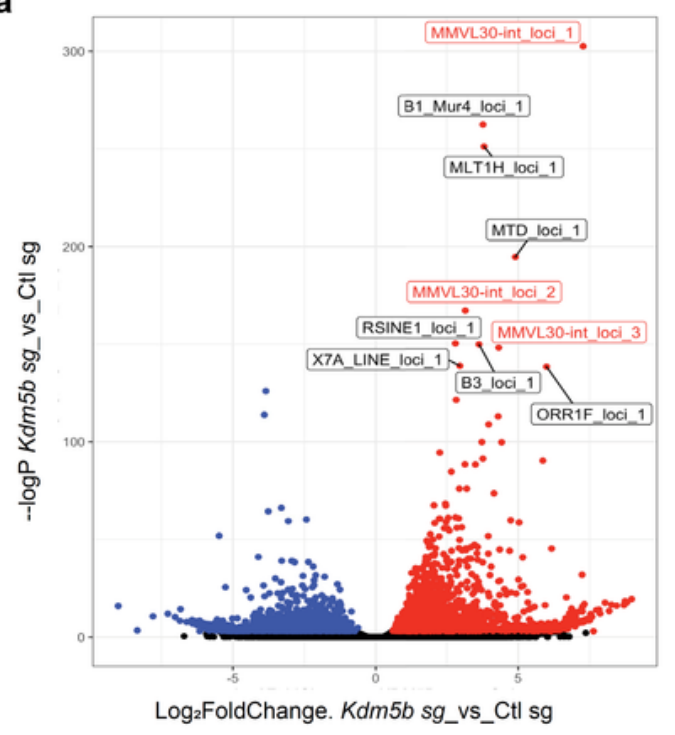

b

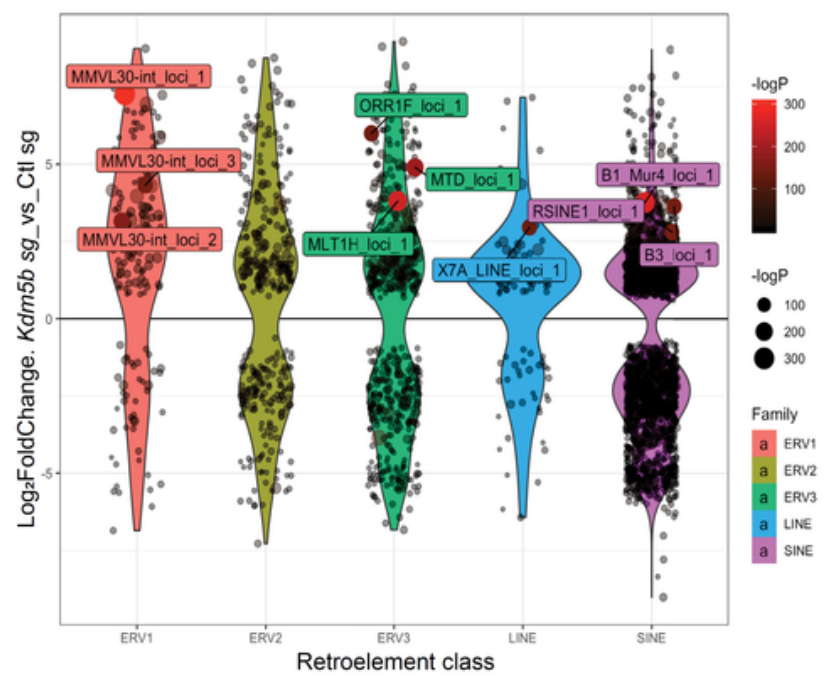

d

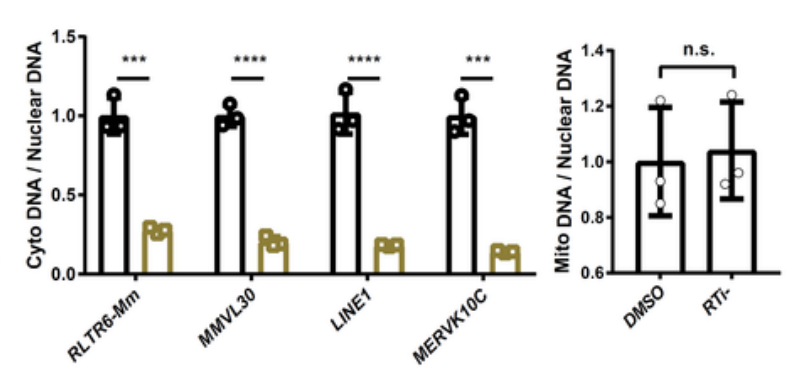

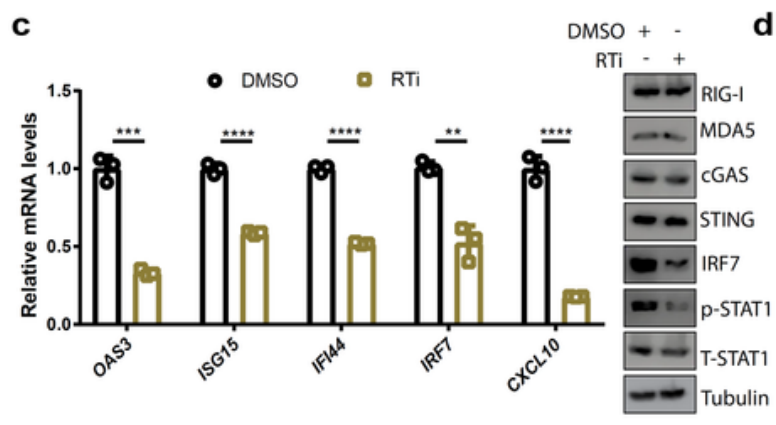

f

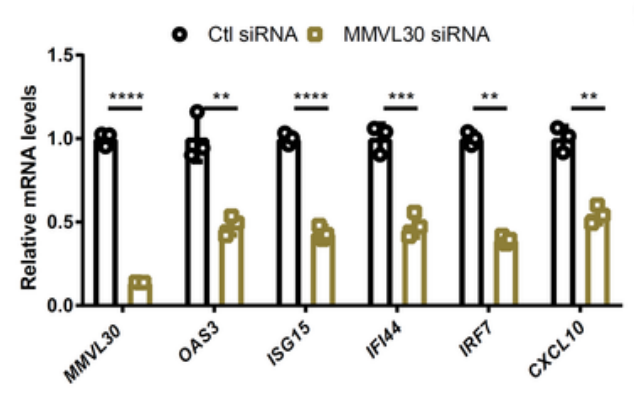

g

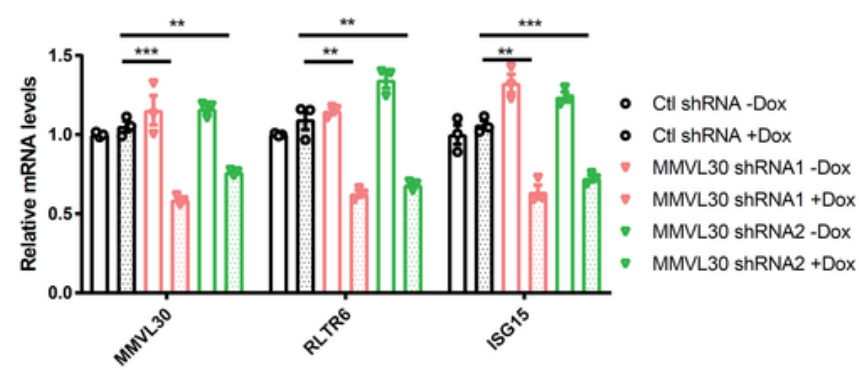

\section{Figure 3}

Deletion of KDM5B de-represses endogenous retroelements, which can be reverse transcribed into cDNAs, activating both RNA and DNA sensing pathways. a. Differential retroelements transcript analysis of Kdm5b sg vs control YUMMER1.7 RNA-seq data shown in volcano blots, combining both forward and reverse strand transcripts. Dots in red represent increased expression of retroelements $(\log 2(\mathrm{FC})>0$ and FDR< 0.05) and Dots in blue represent decreased expression of transposable elements $(\log 2(F C)<0$ and 
FDR 0.05 ). MMVL30-int transcripts are highlighted in red. b, Violin plots showing differential expressed retroelement classes comparing Kdm5b sg to control YUMMER1.7 cells (FDR<0.05). c, RT-qPCR analyses of ISGs in Kdm5b sg YUMMER1.7 cells treated with $20 \mu \mathrm{M}$ of reverse transcriptase inhibitors (RTi) for 48h. Right: Western blot analyses of DNA and RNA sensors, as well as interferon signaling proteins in the cells used in c. Data are represented as mean \pm SD. d, qPCR analyses of retroelements from DNA isolated from cytosolic (cyto) lysate versus from nuclear lysate. Data are represented as mean $\pm S D$. e, Relative copy number of mitochondrial (mito) DNA quantified by qPCR analyses with Dloop primers. Data are represented as mean \pm SD. f, RT-qPCR analyses of MMVL30 and ISGs in Kdm5b-/-cells treated with control or MMVL30 targeting siRNA for 3 days. Data are represented as mean \pm SD. g, RT-qPCR analyses of MMVL30, RLTR6 and ISG15 in Kdm5b-/- cells with the indicated doxycycline (Dox) inducible shRNAs. Data are represented as mean \pm SEM. All statistic $p$ values for $c$ and $d-g$ were calculated using unpaired

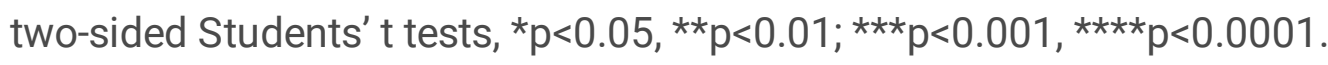


Figure 3.

a

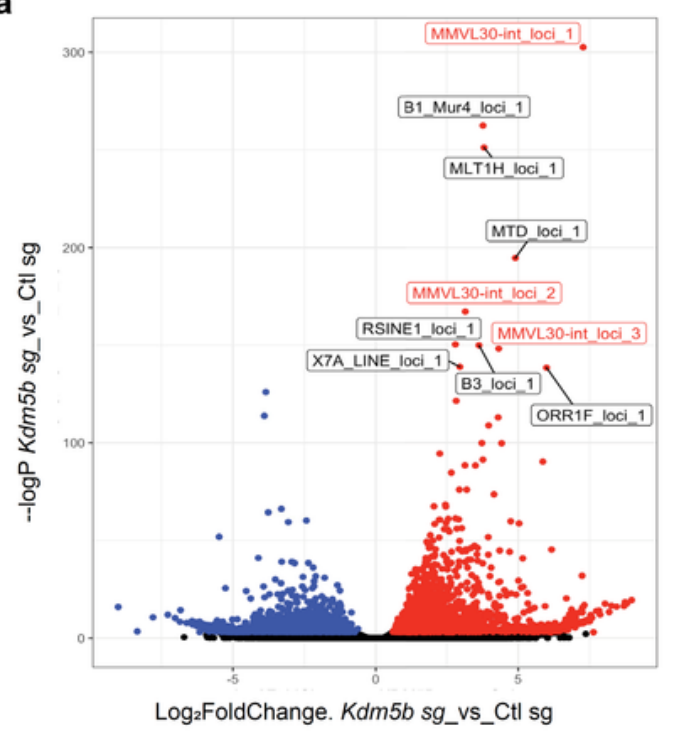

b

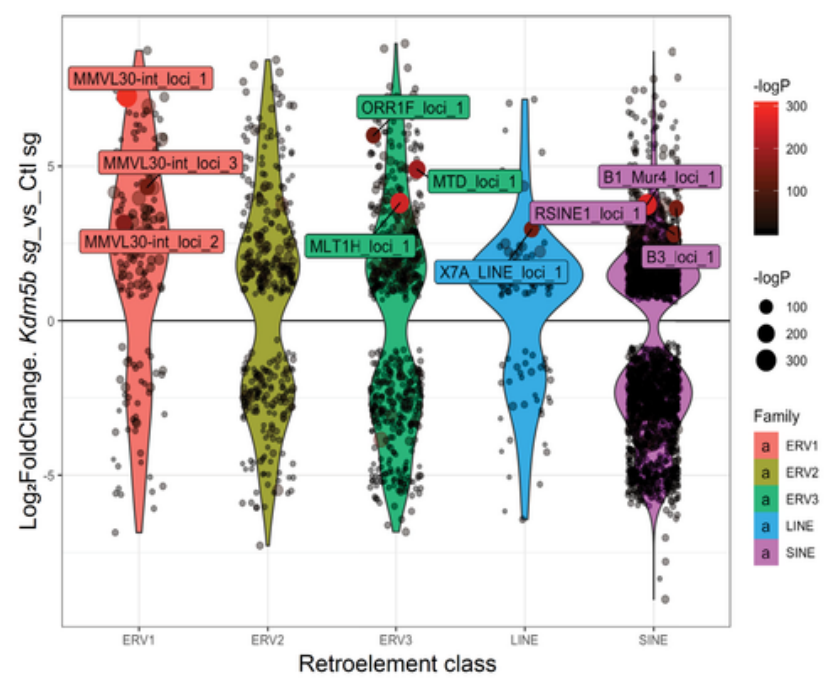

d





f

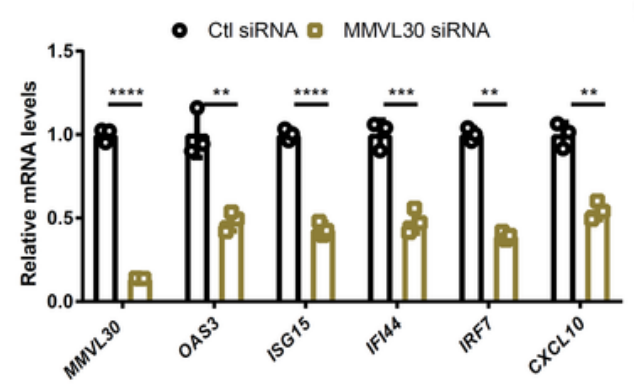

g

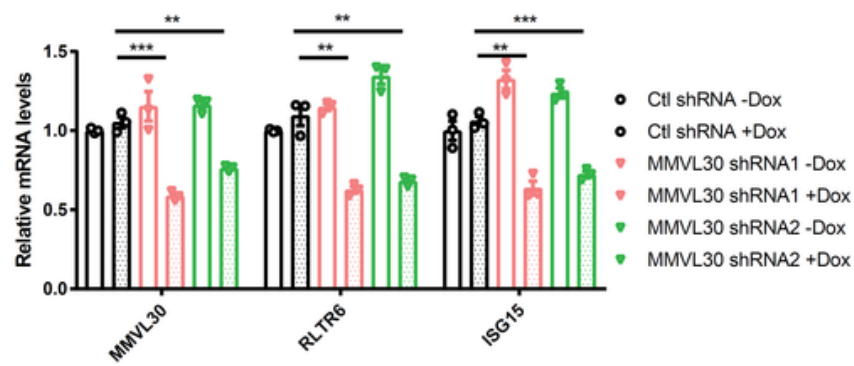

\section{Figure 3}

Deletion of KDM5B de-represses endogenous retroelements, which can be reverse transcribed into cDNAs, activating both RNA and DNA sensing pathways. a. Differential retroelements transcript analysis of Kdm5b sg vs control YUMMER1.7 RNA-seq data shown in volcano blots, combining both forward and reverse strand transcripts. Dots in red represent increased expression of retroelements $(\log 2(\mathrm{FC})>0$ and FDR< 0.05) and Dots in blue represent decreased expression of transposable elements $(\log 2(F C)<0$ and 
FDR< 0.05). MMVL30-int transcripts are highlighted in red. b, Violin plots showing differential expressed retroelement classes comparing Kdm5b sg to control YUMMER1.7 cells (FDR<0.05). c, RT-qPCR analyses of ISGs in Kdm5b sg YUMMER1.7 cells treated with $20 \mu \mathrm{M}$ of reverse transcriptase inhibitors (RTi) for 48h. Right: Western blot analyses of DNA and RNA sensors, as well as interferon signaling proteins in the cells used in c. Data are represented as mean \pm SD. d, qPCR analyses of retroelements from DNA isolated from cytosolic (cyto) lysate versus from nuclear lysate. Data are represented as mean \pm SD. e, Relative copy number of mitochondrial (mito) DNA quantified by qPCR analyses with Dloop primers. Data are represented as mean \pm SD. f, RT-qPCR analyses of MMVL30 and ISGs in Kdm5b-/-cells treated with control or MMVL30 targeting siRNA for 3 days. Data are represented as mean \pm SD. g, RT-qPCR analyses of MMVL30, RLTR6 and ISG15 in Kdm5b-/- cells with the indicated doxycycline (Dox) inducible shRNAs. Data are represented as mean \pm SEM. All statistic $p$ values for $c$ and $d-g$ were calculated using unpaired two-sided Students' t tests, ${ }^{*} p<0.05,{ }^{* \star} p<0.01 ;{ }^{\star \star *} \mathrm{p}<0.001,{ }^{\star \star \star \star *} \mathrm{p}<0.0001$.

\section{Figure 4}

KDM5B suppresses the expression of retroelements by recruiting H3K9 methyltransferase SETDB1. a-b, ChIP-qPCR analyses of WT and Kdm5b-/-YUMMER1.7 cells. Data are represented as mean \pm SEM. c, RTqPCR analyses of the indicated retroelements and ISGs in Kdm5b-/- YUMMER1.7 cells, with control GFP, or reconstituted expression of WT-KDM5B or enzymatic dead mutant (MT) KDM5B. Data are represented as mean \pm SEM.d, Tumor growth curves of WT C57BL/ 6 mice injected with the cells used in $c,(n=6)$. Data are represented as mean \pm SEM. e, ChIP-qPCR analyses of WT and Kdm5b-/-YUMMER1.7 cells. Data are represented as mean \pm SEM. f, ChIP-qPCR analyses of WT YUMMER cells with GFP, Kdm5b-/- YUMMER1.7 cells, with control GFP, or reconstituted expression of WT-KDM5B or enzymatic dead mutant (MT) KDM5B. Data are represented as mean \pm SEM. g, Western blot analyses of SETDB1 immunoprecipitates (IP) with nuclear extracts from control (Ctl), Kdm5b sg and Setdb1 sg YUMMER1.7 cells. h, A working model showing that KDM5B suppresses anti-tumor responses by recruiting SETDB1 to inhibit endogenous retroelements and type I interferon response. $p$ values for $a-c$, and $e-f$ were calculated using unpaired two-sided Students' t tests, n.s. no significant difference, ${ }^{\star} p<0.05 ;{ }^{* *} p<0.01 ; * \star \star p<0.001$, $\star \star \star \star p<0.0001$. 2-way ANOVA analysis was utilized for comparison at day 16 in $d,{ }^{\star} * \star \star x<0.0001$. 
Figure 4.
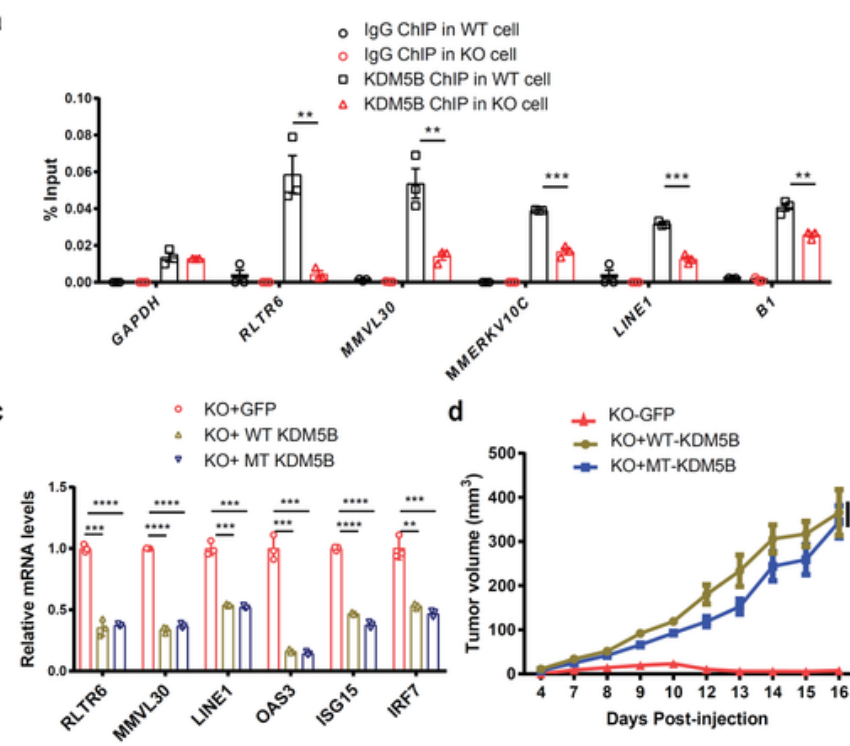$$
\text { SETDB1 ChIP 口 WT+GFP }
$$

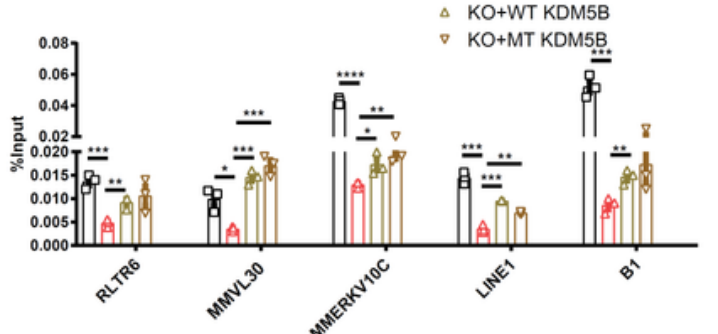

b

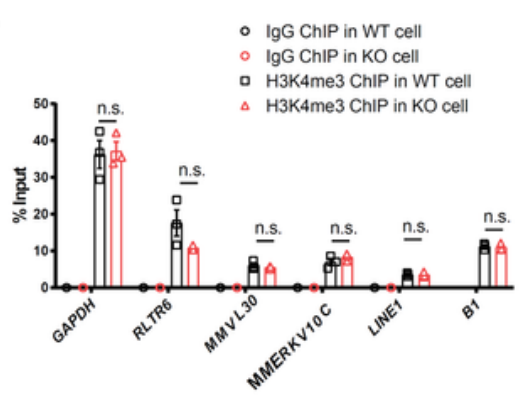

e

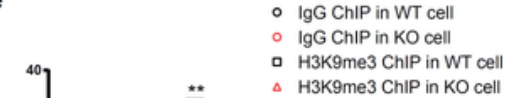

${ }^{40}{ }^{4} \quad$ a $\mathrm{H}_{3 \mathrm{~K} 9 \mathrm{me}} 3 \mathrm{ChIP}$ in WT cell

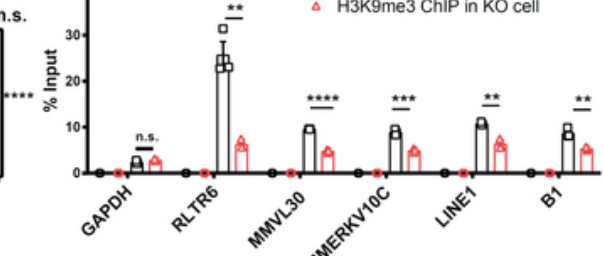

h
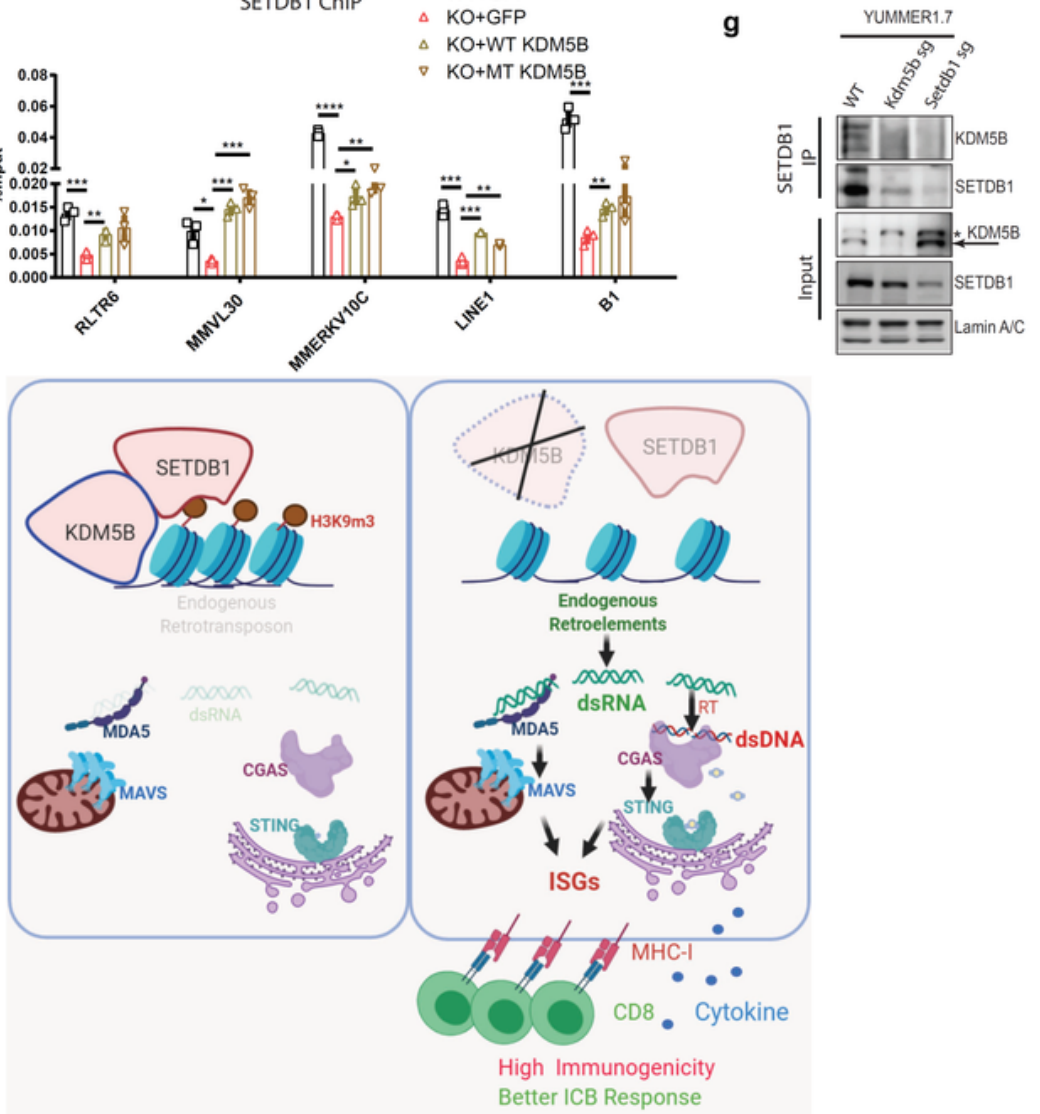

\section{Figure 4}

KDM5B suppresses the expression of retroelements by recruiting H3K9 methyltransferase SETDB1. $a-b$, ChIP-qPCR analyses of WT and Kdm5b-/-YUMMER1.7 cells. Data are represented as mean \pm SEM. c, RTqPCR analyses of the indicated retroelements and ISGs in Kdm5b-/- YUMMER1.7 cells, with control GFP, or reconstituted expression of WT-KDM5B or enzymatic dead mutant (MT) KDM5B. Data are represented as mean \pm SEM.d, Tumor growth curves of WT C57BL/6 mice injected with the cells used in $c,(n=6)$. Data 
are represented as mean \pm SEM. e, ChIP-qPCR analyses of WT and Kdm5b-/-YUMMER1.7 cells. Data are represented as mean \pm SEM. f, ChIP-qPCR analyses of WT YUMMER cells with GFP, Kdm5b-/- YUMMER1.7 cells, with control GFP, or reconstituted expression of WT-KDM5B or enzymatic dead mutant (MT) KDM5B. Data are represented as mean \pm SEM. $g$, Western blot analyses of SETDB1 immunoprecipitates (IP) with nuclear extracts from control (Ctl), Kdm5b sg and Setdb1 sg YUMMER1.7 cells. h, A working model showing that KDM5B suppresses anti-tumor responses by recruiting SETDB1 to inhibit endogenous retroelements and type I interferon response. $p$ values for $a-c$, and e-f were calculated using unpaired two-sided Students' $t$ tests, n.s. no significant difference, ${ }^{*} p<0.05 ;{ }^{* \star} p<0.01 ;{ }^{* \star *} p<0.001$, $\star \star \star \star p<0.0001$. 2-way ANOVA analysis was utilized for comparison at day 16 in $d,{ }^{\star} * \star \star p<0.0001$.

\section{Supplementary Files}

This is a list of supplementary files associated with this preprint. Click to download.

- ExtendedDataFigurelegend100420.pdf

- ExtendedDataFigurelegend100420.pdf

- ExtendedDataTables210100420.xIsx

- ExtendedDataTables210100420.xlsx 